\title{
Dynamical Collapse of Charged Scalar Field in Phantom Gravity
}

\author{
Anna Nakonieczna and Marek Rogatko \\ Institute of Physics \\ Maria Curie-Sklodowska University \\ 20-031 Lublin, pl. Marii Curie-Sklodowskiej 1, Poland \\ aborkow@kft.umcs.lublin.pl \\ rogat@kft.umcs.lublin.pl \\ marek.rogatko@poczta.umcs.lublin.pl \\ Rafał Moderski \\ Nicolaus Copernicus Astronomical Center \\ Polish Academy of Sciences \\ 00-716 Warsaw, Bartycka 18, Poland \\ moderski@camk.edu.pl
}

(Dated: June 4, 2021)

\begin{abstract}
We investigated the problem of the dynamical collapse of a self-gravitating complex charged scalar field in Einstein-Maxwell-dilaton theory with a phantom coupling for the adequate fields in the system under consideration. We also considered two simplifications of it, i.e., the separate collapses of phantom Maxwell and phantom scalar fields under the influence of Einstein gravity. One starts with the regular spacetime and leads the evolution through the formation of the horizons and the final singularity. We discuss the structures of spacetimes emerging in the process of the dynamical collapse and comment on the role of the considered fields in its course.
\end{abstract}

PACS numbers: 04.25.dg, 04.40.-b

\section{INTRODUCTION}

Phantom gravitating fields emerge with a kinetic term with the wrong sign. It turns out that this fact implies that they are coupled repulsively to gravity. These fields could form ghost condensation at the quantum level, which in turn might lead to the modification of gravity at the infrared limit [1. On the other hand, the present observations of the Universe reveal the fact that it may be dominated by an exotic kind of fields with negative pressure causing the acceleration. Type Ia supernovae (Sn Ia) provide a precise calibrated standard candle with which one can probe the expansion of the Universe on large scales. In the late 1990s the independent surveys for distant Sn Ia announced that the high-redshifted supernovae of this type appeared about 40 percent fainter or equivalently more distant than expected in a flat, matter-dominated Universe [2. These researches were combined with other concerning the composition of the Universe such as cosmic microwave background (CMB) observations [3] or analyzes of barionic acoustic oscillations (BAO) [4]. Altogether they do not exclude the possibility that the exotic matter mentioned above consists of the fields with the so-called super-negative pressure, i.e., when $p<-\rho$ ( $p$ and $\rho$ stand for the pressure and density of matter, respectively) [5. Such fields are dubbed phantom.

The presence of this kind of fields which contribute negatively to the total energy, i.e., when the violation of the null energy condition takes place, gives rise to the interesting possibilities of new solutions of Einstein equations coupled to phantom field. Namely, in Ref. [6] the wormhole solutions with phantom scalar field were studied both analytically and numerically. Phantom field has also a direct consequence for black hole physics. In Ref. [7] in theories where gravity coupled to Maxwell and dilaton fields in such a way that the kinetic energy for either or both of the fields in question was allowed to be negative, black hole solutions were obtained. Among all it was found that regular black hole solutions with zero or negative mass could be provided. On the other hand, cold black holes with a multiply degenerate event horizon and an infinite horizon area were considered in [8]. The new class of static, spherically symmetric solutions in Einstein-Maxwell-dilaton theory with phantom coupling for the dilaton and/or Maxwell field leading to black holes with single or multiple horizons were elaborated in Ref. [9]. The static multicenter solutions of phantom Einstein-Maxwell-dilaton theory and gravitating $\sigma$-models obtained via dimensional reduction of phantom Einstein-Maxwell, phantom Kaluza-Klein and phantom Einstein-Maxwell-dilaton-axion theories were discussed in [10.

On the other hand, the gravitational collapse and the no-hair conjecture or its mathematical formulation black hole uniqueness theorem, stating that stationary axisymmetric solution of Einstein-Maxwell equations relaxes to KerrNewman spacetime and is characterized by black hole mass, charge and angular momentum [11, attracted attention for many decades of researches. A lot of progress has been also made during last years to answer the basic question underlying the theoretical studies of black hole interiors. Quite different description of the inner black hole singularity 
emerged [12]-14]. A full nonlinear description of the inner structure of black holes was presented in Ref.[15]. Then, in [16] the spherically symmetric collapse of massless scalar field was numerically examined. It was shown that the field dispersed to infinity or collapsed to a black hole. The aforementioned researches were also broaden to the case of the nonlinear evolution of the neutral scalar field on a background spacetime of charged black hole [17. Ref. [18] was devoted to the numerical solution of spherically symmetric semi-classical scalar field collapse. The addition of the effective energy momentum tensor describing evaporation of black hole enabled to consider the influence of this process on a critical phenomena present in classical collapse. In [19] the occurrence of the mass inflation phenomenon during a dynamical charged gravitational collapse was shown. As far as mass inflation is concerned, it was shown in 14 that in a realistic RN black hole the Cauchy horizon was unstable due to the fact that it developed a weak null singularity caused by the local mass parameter growing indefinitely large.

The case of the dynamical collapse of spherically symmetric shell of the charged massless scalar field was elaborated in Ref.[20. An external RN spacetime and the inner spacetime bounded by singularity on the Cauchy horizon were obtained from numerical calculations. In order to figure out pair creation process in strong electric field in the dynamical collapse of electrically charged massless scalar field, the dynamical formation of evaporating spherically symmetric charged black hole emitting Hawking radiation and the behaviour of complex scalar field, gauge field and the normalized energy momentum tensor during the process in question, numerical approaches were performed [21]24. These researches were supplemented by the studies of the behaviour of Brans-Dicke field 25] during gravitational collapse of matter.

Apart from studying the full gravitational collapse on flat background spacetime, the wide range of simulations of an accretion onto an existing black hole was also carried out. The most interesting for us exotic matter accreting onto the Schwarzschild [26] or Reissner-Nordström black hole [27] was modeled as a free scalar field with the opposite sign in its energy momentum tensor.

Because of the fact that the uniqueness theorem for the low-energy string black holes is quite well established [28, the implication of the superstring theory on the dynamical gravitational collapse of charged scalar field was studied in [29. Numerical studies revealed that the dynamical collapse in question led to the formation of Schwarzschild-like spacetimes in case of non-zero coupling between the charged scalar field and the fields emerging from the superstring theory.

As was mentioned above, the presence of phantom fields as well as phantom coupling of the ordinary fields, gives rise to new possibilities for solutions of generalized Einstein equations. Motivated by the aforementioned analytical and numerical researches we shall consider the problem of the full nonlinear dynamical collapse of a complex scalar field when gravitational interactions take forms of Einstein-phantom Maxwell (E $\bar{M})$, Einstein-phantom Maxwell-dilaton (E $\bar{M} D)$, Einstein-Maxwell-phantom dilaton $(E M \bar{D})$, and Einstein-phantom Maxwell-phantom dilaton $(\mathrm{E} \overline{M D})$ theories. We will also consider the evolution in Einstein-phantom dilaton $(\mathrm{E} \bar{D})$ theory, namely the collapse of phantom scalar field in the regime of Einstein gravity.

Our paper is organized as follows. In Sec.II we derive equations of motion describing the gravitational collapse of a shell of charged scalar field in Einstein-Maxwell-dilaton theory with phantom coupling. Sec.III is connected with the numerical scheme applied in our investigations. In Sec.IV we discuss obtained results, while in Sec.V we present the conclusions of our investigations.

\section{PHANTOM EINSTEIN-MAXWELL-DILATON THEORY}

The main aim of our researches will be to study the dynamical collapse of a complex scalar field in the stringinspired background with phantom coupling of the fields in the theory. Because of the fact that the coupling constant of dilaton field to the complex scalar field is unknown, the considered action is provided in the form written in the string frame as follows:

$$
\hat{I}=\int d^{4} x \sqrt{-\hat{g}}\left[e^{-2 \phi}\left(\hat{R}-2 \xi_{1}(\hat{\nabla} \phi)^{2}+e^{2 \alpha \phi} \mathcal{L}\right)\right],
$$

where $\phi$ denotes the dilaton field, $\alpha$ stands for the coupling constant between dilaton and charged scalar field $\psi$ described by the Lagrangian $\mathcal{L}$ of the form as

$$
\mathcal{L}=-\frac{1}{2}\left(\hat{\nabla}_{\alpha} \psi+i e A_{\alpha} \psi\right) \hat{g}^{\alpha \beta}\left(\hat{\nabla}_{\beta} \psi^{*}-i e A_{\beta} \psi^{*}\right)-\xi_{2} F_{\mu \nu} F^{\mu \nu} .
$$

$\xi_{1}$ and $\xi_{2}$ are dilaton-gravity and $U(1)$-gauge field-gravity coupling constants. In the normal case of Einstein-Maxwelldilaton-complex scalar field system they are equal to 1 . On the contrary, in phantom case we have $\xi_{1}=-1$ and/or 
$\xi_{2}=-1$. After performing the conformal transformation, the action may be rewritten in the so-called Einstein frame. It yields

$$
I=\int d^{4} x \sqrt{-g}\left[R-2 \xi_{1}(\nabla \phi)^{2}+e^{2 \alpha \phi+4 \phi} \mathcal{L}\left(\psi, \psi^{*}, A, e^{2 \phi} g_{\alpha \beta}\right)\right]
$$

Taking variations with respect to the adequate fields in the theory in question, we obtain the following equations of motion:

$$
\begin{aligned}
\nabla^{2} \phi & -\frac{\alpha+1}{4 \xi_{1}} e^{2 \phi(\alpha+1)}\left(\nabla_{\beta} \psi+i e A_{\beta} \psi\right)\left(\nabla^{\beta} \psi^{*}-i e A^{\beta} \psi^{*}\right)-\frac{1}{2} \frac{\xi_{2}}{\xi_{1}} \alpha e^{2 \alpha \phi} F^{2}=0 \\
\nabla_{\mu}\left(e^{2 \alpha \phi} F^{\mu \nu}\right) & +\frac{e^{2 \phi(\alpha+1)}}{4 \xi_{2}}\left[i e \psi^{*}\left(\nabla^{\nu} \psi+i e A^{\nu} \psi\right)-i e \psi\left(\nabla^{\nu} \psi^{*}-i e A^{\nu} \psi^{*}\right)\right]=0 \\
\nabla^{2} \psi & +i e A^{\beta}\left(2 \nabla_{\beta} \psi+i e A_{\beta} \psi\right)+i e \nabla_{\delta} A^{\delta} \psi=0 \\
\nabla^{2} \psi^{*} & -i e A^{\beta}\left(2 \nabla_{\beta} \psi^{*}-i e A_{\beta} \psi^{*}\right)-i e \nabla_{\delta} A^{\delta} \psi^{*}=0 \\
G_{\mu \nu} & =T_{\mu \nu}\left(\phi, F, \psi, \psi^{*}, A\right)
\end{aligned}
$$

The energy momentum tensor $T_{\mu \nu}\left(\phi, F, \psi, \psi^{*}, A\right)$ is given by

$$
T_{\mu \nu}\left(\phi, F, \psi, \psi^{*}, A\right)=e^{2 \phi(\alpha+1)} \tilde{T}_{\mu \nu}\left(\psi, \psi^{*}, A\right)+T_{\mu \nu}(F, \phi),
$$

where $\tilde{T}_{\mu \nu}\left(\psi, \psi^{*}, A\right)$ implies

$$
\begin{aligned}
\tilde{T}_{\mu \nu}\left(\psi, \psi^{*}, A\right) & =\frac{1}{4}\left[i e \psi\left(A_{\mu} \nabla_{\nu} \psi^{*}+A_{\nu} \nabla_{\mu} \psi^{*}\right)-i e \psi^{*}\left(A_{\mu} \nabla_{\nu} \psi+A_{\nu} \nabla_{\mu} \psi\right)\right] \\
& +\frac{1}{4}\left(\nabla_{\mu} \psi \nabla_{\nu} \psi^{*}+\nabla_{\mu} \psi^{*} \nabla_{\nu} \psi\right)+\frac{1}{2} e^{2} A_{\mu} A_{\nu} \psi \psi^{*}+\frac{1}{2} \tilde{\mathcal{L}}\left(\psi, \psi^{*}, A\right) g_{\mu \nu}
\end{aligned}
$$

On the other hand, the explicit form of the Lagrangian $\tilde{\mathcal{L}}\left(\psi, \psi^{*}, A\right)$ is provided by the expression

$$
\tilde{\mathcal{L}}\left(\psi, \psi^{*}, A\right)=-\frac{1}{2}\left(\nabla_{\beta} \psi+i e A_{\beta} \psi\right)\left(\nabla^{\beta} \psi^{*}-i e A^{\beta} \psi^{*}\right) .
$$

The energy momentum tensor for $U(1)$-gauge and dilaton fields yields

$$
T_{\mu \nu}(F, \phi)=\xi_{2} e^{2 \alpha \phi}\left(2 F_{\mu \rho} F_{\nu}{ }^{\rho}-\frac{1}{2} g_{\mu \nu} F^{2}\right)+\xi_{1}\left(2 \nabla_{\mu} \phi \nabla_{\nu} \phi-g_{\mu \nu}(\nabla \phi)^{2}\right) .
$$

In our research we shall start with the regular initial spacetime at approximately past null infinity, compute the formation of the horizons and follow the evolution of the dynamically formed black hole to the central singularity. One chooses $(2+2)$-spherically symmetric double null coordinate system. It turned out that any coordinate gauge transformation of the form $u \rightarrow f(u)$ and $v \rightarrow g(v)$ will preserve the null character of the retarded and advanced coordinates. The line element written in the aforementioned coordinates is provided by [30]:

$$
d s^{2}=-a(u, v)^{2} d u d v+r^{2}(u, v) d \Omega^{2} .
$$

The assumptions about spherical symmetry of the problem imply that the only non-vanishing components of the $U(1)$-gauge strength field are $F_{u v}$ and $F_{v u}$. Moreover, another restriction on the gauge potential can be implemented. We can consider $A_{u}$ or $A_{v}$. It turned out [29] that one can restrict his attention to the only one gauge potential due to the fact of the gauge freedom $A_{u} \rightarrow A_{u}+\nabla_{u} \theta$, where $\theta=\int A_{v} d v$. Consequently, we are left with the only one component of the gauge field being the function of the retarded and advanced coordinates.

Defining the quantity like in Ref.[20]:

$$
Q=2 \frac{A_{u, v} r^{2}}{a^{2}}
$$


enables us to separate $v$-component of the second order partial differential generalized Einstein-Maxwell equations (5) given by

$$
\left[\frac{2 e^{2 \alpha \phi} r^{2} A_{u, v}}{a^{2}}\right]_{, v}+\frac{r^{2} e^{2 \phi(\alpha+1)}}{4 \xi_{2}} i e\left(\psi^{*} \psi_{, v}-\psi \psi_{, v}^{*}\right)=0
$$

into much simpler first order differential equations for $A_{u}$

$$
A_{u, v}-\frac{Q a^{2}}{2 r^{2}}=0
$$

and for $Q$, which in turn yields

$$
Q_{, v}+2 \alpha \phi_{, v} Q+\frac{i e r^{2}}{4 \xi_{2}} e^{2 \phi}\left(\psi^{*} \psi_{, v}-\psi \psi_{, v}^{*}\right)=0
$$

On the other hand, Eq. 4 has the explicit form as follows:

$$
r_{, u} \phi_{, v}+r_{, v} \phi_{, u}+r \phi_{, u v}-\frac{(\alpha+1)}{8 \xi_{1}} e^{2 \phi(\alpha+1)} r\left[\psi_{, u} \psi_{, v}^{*}+\psi_{, v} \psi_{, u}^{*}+i e A_{u}\left(\psi \psi_{, v}^{*}-\psi^{*} \psi_{, v}\right)\right]-\alpha \frac{\xi_{2}}{\xi_{1}} \frac{a^{2} Q^{2}}{4 r^{3}}=0 .
$$

An inspection of the relations for the complex scalar field (6)-(7) reveals that one gets the following:

$$
\begin{gathered}
r_{, u} \psi_{, v}+r_{, v} \psi_{, u}+r \psi_{, u v}+i e r A_{u} \psi_{, v}+i e r_{, v} A_{u} \psi+\frac{i e Q a^{2}}{4 r} \psi=0 \\
r_{, u} \psi_{, v}^{*}+r_{, v} \psi_{, u}^{*}+r \psi_{, u v}^{*}-i e r A_{u} \psi_{, v}^{*}-i e r_{, v} A_{u} \psi^{*}-\frac{i e Q a^{2}}{4 r} \psi^{*}=0 .
\end{gathered}
$$

Furthermore, the adequate components of the Einstein tensor and the stress-energy tensor for the underlying theory reveal the set of equations

$$
\begin{aligned}
\frac{2 a_{, u} r_{, u}}{a}-r_{, u u} & =\xi_{1} r \phi_{, u}^{2}+\frac{r e^{2 \phi(\alpha+1)}}{4}\left[\psi_{, u} \psi_{, u}^{*}+i e A_{u}\left(\psi \psi_{, u}^{*}-\psi^{*} \psi_{, u}\right)+e^{2} A_{u}^{2} \psi \psi^{*}\right], \\
\frac{2 a_{, v} r_{, v}}{a}-r_{, v v} & =\xi_{1} r \phi_{v}^{2}+\frac{1}{4} r e^{2 \phi(\alpha+1)} \psi_{, v} \psi_{, v}^{*} \\
\frac{a^{2}}{4 r}+\frac{r_{, u} r_{v}}{r}+r_{, u v} & =\xi_{2} \frac{e^{2 \alpha \phi} a^{2} Q^{2}}{4 r^{3}} \\
\frac{a_{, u} a_{, v}}{a^{2}}-\frac{a_{, u v}}{a}-\frac{r_{, u v}}{r} & =\xi_{2} \frac{Q^{2} e^{2 \alpha \phi} a^{2}}{4 r^{4}}+\xi_{1} \phi_{, u} \phi_{, v}+\frac{e^{2 \phi(\alpha+1)}}{8}\left[\psi_{, u} \psi_{, v}^{*}+\psi_{, u}^{*} \psi_{, v}+i e A_{u}\left(\psi \psi_{v}^{*}-\psi^{*} \psi_{, v}\right)\right] .
\end{aligned}
$$

To proceed further, one introduces the new auxiliary variables written in the following forms:

$$
\begin{array}{rrrr}
c=\frac{a_{, u}}{a}, & d=\frac{a_{, v}}{a}, & f=r_{, u}, & g=r_{, v}, \\
s=\psi, & p=\psi_{, u}, & q=\psi_{, v}, & \beta=A_{u}, \\
k=\phi, & x=\phi_{, u}, & y=\phi_{, v} .
\end{array}
$$

Moreover, the additional quantities take the forms as

$$
\begin{aligned}
\lambda & \equiv \frac{a^{2}}{4}+f g, \\
\mu & \equiv f q+g p, \\
\eta & \equiv g x+f y .
\end{aligned}
$$

It can be easily seen that instead of taking into account two complex fields $\psi$ and $\psi^{*}$, one can introduce two real fields. Namely, they satisfy the relations $\psi=\psi_{1}+i \psi_{2}$ and $\psi^{*}=\psi_{1}-i \psi_{2}$. Under these assumptions we arrive at

$$
\begin{aligned}
& s=s_{1}+i s_{2}, \quad p=p_{1}+i p_{2}, \quad q=q_{1}+i q_{2}, \\
& \mu=\mu_{1}+i \mu_{2}, \quad \mu_{1}=f q_{1}+g p_{1}, \quad \mu_{2}=f q_{2}+g p_{2} \text {. }
\end{aligned}
$$


Just, the system of the second order partial differential equations can be rewritten in the form of the first order one. It can be checked that we finally arrive at the set of the first order differential equations written as follows:

$$
\begin{aligned}
& \text { P1 : } \quad a_{, u}-a c=0, \\
& P 2: \quad a_{, v}-a d=0, \\
& \text { P3 : } \quad r_{, u}-f=0, \\
& \text { P4: } \quad r_{, v}-g=0, \\
& P 5_{(R e)}: s_{1, u}-p_{1}=0 \text {, } \\
& P 5_{(I m)}: s_{2, u}-p_{2}=0 \text {, } \\
& P 6_{(\text {Re })}: s_{1, v}-q_{1}=0 \text {, } \\
& P 6_{(I m)}: s_{2, v}-q_{2}=0 \text {, } \\
& \text { P7 : } \quad k_{, u}-x=0, \\
& \text { P8 : } \quad k_{, v}-y=0 \text {, } \\
& E 1: \quad f_{, u}-2 c f+\xi_{1} r x^{2}+\frac{1}{4} r e^{2 k(\alpha+1)}\left[p_{1}^{2}+p_{2}^{2}+2 e \beta\left(s_{1} p_{2}-s_{2} p_{1}\right)+e^{2} \beta^{2}\left(s_{1}^{2}+s_{2}^{2}\right)\right]=0 \\
& E 2: \quad g_{, v}-2 d g+\xi_{1} r y^{2}+\frac{1}{4} r e^{2 k(\alpha+1)}\left(q_{1}^{2}+q_{2}^{2}\right)=0, \\
& E 3^{(1)}: f_{, v}+\frac{\lambda}{r}-\xi_{2} e^{2 \alpha k} \frac{Q^{2} a^{2}}{4 r^{3}}=0 \\
& E 3^{(2)}: g_{, u}+\frac{\lambda}{r}-\xi_{2} e^{2 \alpha k} \frac{Q^{2} a^{2}}{4 r^{3}}=0 \\
& E 4^{(1)}: c_{, v}-\frac{\lambda}{r^{2}}+\xi_{1} x y+\frac{1}{4} e^{2 k(\alpha+1)}\left[p_{1} q_{1}+p_{2} q_{2}+e \beta\left(s_{1} q_{2}-s_{2} q_{1}\right)\right]+\xi_{2} e^{2 \alpha k} \frac{Q^{2} a^{2}}{2 r^{4}}=0 \\
& E 4^{(2)}: d_{, u}-\frac{\lambda}{r^{2}}+\xi_{1} x y+\frac{1}{4} e^{2 k(\alpha+1)}\left[p_{1} q_{1}+p_{2} q_{2}+e \beta\left(s_{1} q_{2}-s_{2} q_{1}\right)\right]+\xi_{2} e^{2 \alpha k} \frac{Q^{2} a^{2}}{2 r^{4}}=0 \\
& S_{(R e)}^{(1)}: \quad r p_{1, v}+\mu_{1}-e r \beta q_{2}-e s_{2} \beta g-e s_{2} \frac{Q a^{2}}{4 r}=0, \\
& S_{(I m)}^{(1)}: r p_{2, v}+\mu_{2}+e r \beta q_{1}+e s_{1} \beta g+e s_{1} \frac{Q a^{2}}{4 r}=0, \\
& S_{(R e)}^{(2)}: r q_{1, u}+\mu_{1}-e r \beta q_{2}-e s_{2} \beta g-e s_{2} \frac{Q a^{2}}{4 r}=0, \\
& S_{(I m)}^{(2)}: r q_{2, u}+\mu_{2}+e r \beta q_{1}+e s_{1} \beta g+e s_{1} \frac{Q a^{2}}{4 r}=0, \\
& D^{(1)}: \quad r x_{, v}+\eta-\frac{\alpha+1}{4 \xi_{1}} r e^{2 k(\alpha+1)}\left[p_{1} q_{1}+p_{2} q_{2}+e \beta\left(s_{1} q_{2}-s_{2} q_{1}\right)\right]-\alpha \frac{\xi_{2}}{\xi_{1}} e^{2 \alpha k} \frac{Q^{2} a^{2}}{4 r^{3}}=0 \\
& D^{(2)}: \quad r y_{, u}+\eta-\frac{\alpha+1}{4 \xi_{1}} r e^{2 k(\alpha+1)}\left[p_{1} q_{1}+p_{2} q_{2}+e \beta\left(s_{1} q_{2}-s_{2} q_{1}\right)\right]-\alpha \frac{\xi_{2}}{\xi_{1}} e^{2 \alpha k} \frac{Q^{2} a^{2}}{4 r^{3}}=0 \\
& M 1: \quad \beta_{, v}-\frac{Q a^{2}}{2 r^{2}}=0, \\
& \text { M2: } \quad Q_{, v}+2 \alpha y Q-\frac{1}{2 \xi_{2}} e^{2 k} \text { e } r^{2}\left(s_{1} q_{2}-s_{2} q_{1}\right)=0 \text {. }
\end{aligned}
$$

Let us introduce the quantity of the physical interest. Namely, we define the mass function provided by the relation

$$
m(u, v)=\frac{r}{2}\left(1+\frac{4 r_{, u} r_{, v}}{a^{2}}\right)=\frac{r}{2}\left(1+\frac{4}{a^{2}} f g\right) .
$$

It represents the Hawking mass, i.e., the mass included in a sphere of the radius $r(u, v)$.

The characteristics of all the evolutions under consideration are listed in Table I] As was explained in detail in [10] the structures of solutions in $E M D$ as well as in phantom-EMD theories may be regarded as equilibrium states resulting from the competition and/or cooperation among gravitational, electromagnetic and dilatonic forces. The nature of gravitational force is always attractive. The other two forces are either attractive or repulsive depending on whether they are phantom or not. It should be kept in mind that in our case we deal with one more constituent 
TABLE I: Characteristics of evolutions under consideration. The overlining indicates phantom field. Parameters $\tilde{p}_{k}$ and $\tilde{p}_{s}$ denote amplitudes of dilaton and electrically charged scalar fields, respectively.

\begin{tabular}{|c|c|c|c|c|c|c|}
\hline Type of evolution & $\xi_{1}$ & $\xi_{2}$ & $\tilde{p}_{k}$ & $\tilde{p}_{s}$ & $e$ & $\alpha$ \\
\hline$E \bar{M}$ & - & -1 & - & $\neq 0$ & $\neq 0$ & - \\
\hline$E \bar{D}$ & -1 & - & $\neq 0$ & - & - & - \\
\hline$E \bar{M} D$ & +1 & -1 & $\neq 0$ & $\neq 0$ & $\neq 0$ & $0,-1$ \\
\hline$E M \bar{D}$ & -1 & +1 & $\neq 0$ & $\neq 0$ & $\neq 0$ & $0,-1$ \\
\hline$E \overline{M D}$ & -1 & -1 & $\neq 0$ & $\neq 0$ & $\neq 0$ & $0,-1$ \\
\hline
\end{tabular}

of the system under consideration, namely the complex scalar field. Its influence on spacetime structures is uniform in all considered cases, because we do not allow it to be phantom. We shall comment on its role during the collapse after a detailed analysis of spacetime structures.

\section{NUMERICAL COMPUTATIONS}

Unfortunately, a detailed analytical investigations of fully nonlinear gravitational dynamics described by the set of the conjugate equations (31)-(54) are impossible. Therefore, numerical methods ought to be used in order to draw conclusions about the structure of spacetime emerging in the process of the dynamical collapse.

In the present paper we used a numerical algorithm containing an adaptive mesh refinement described in [29]. It was adjusted for the current issue by taking the parameters $\xi_{1}$ and $\xi_{2}$ into account. The manner of setting the boundary and initial conditions for the considered equations remained unchanged in comparison to Ref. 29]. The one-parameter families representing the initial profiles of the field functions used for simulations in the present paper are listed in Table II. The $\left(f_{D}\right)$-family refers to the dilaton field with the family constants $c_{1}=1.3$ and $c_{2}=0.21$. The $\left(f_{S}\right)$-family describes the electrically charged scalar field. The family constant $v_{f}=7.5$ and the parameter determining the amount of the initial electric charge $\delta=\frac{\pi}{2}$ (maximal electric charge) [25]. In both cases the free family parameter is denoted by $\tilde{p}$ with a subscript corresponding to the particular field. Such choice of the initial conditions is physically representative, because the considered collapse is universal, i.e., its course and results do not depend on the types of initial profiles.

There are no analytical solutions neither to the problem in question nor to any of simplified versions of it (apart from the extremely simplified, trivial case of empty spacetime). Thus, checking the correctness of numerical code has to be based on indirect methods.

The three tests used for proving the credibility of the code and justifications for carrying them out, are widely discussed in [29]. As was reported in the aforementioned paper, the results obtained for the different integration steps on the non-adaptive grid display satisfying agreement of an order of $0.01 \%$. Moreover, the code displays the linear convergence as is expected for the applied algorithm and errors decrease with increasing grid density. We reached the same conclusions for the modified code for all possible combinations of $\xi_{1,2}$ shown in Table I. Since the figures depicting them are analogous to the ones presented in the previous paper, we decided not to present them here.

The issue of mass (55) and charge (14) conservation was also analyzed. We obtained profiles of $m$ and $Q$ versus $u$ along ingoing null rays specific to all types of evolutions under consideration. The values of the advanced time were chosen in such a way that the null rays did not intersect any horizons for the small values of the retarded time. The obtained profiles appeared to be in a qualitative agreement with the ones presented in [29]. For the evolutions leading to the formation of a black hole the mass was conserved within $7 \%$ and the electric charge within $4.5 \%$ in regions apart from the vicinities of the apparent horizons. The fluctuations were significant there due to the outgoing fluxes, which appear during the refraction of matter on the potential barriers near the gravitational radius of the collapsing shell [20]. Mass and charge profiles for non-singular spacetimes also displayed expected behaviour. The values tended towards zero for the reason that evolving matter bounced off the non-singular $r=0$ and moved towards future null infinity leaving empty spacetime [16]. 
TABLE II: Initial profiles of field functions.

\begin{tabular}{c|c}
\hline \hline Family & Profile \\
\hline$\left(f_{D}\right)$ & $\tilde{p}_{k} \cdot v^{2} \cdot e^{-\left(\frac{v-c_{1}}{c_{2}}\right)^{2}}$ \\
$\left(f_{S}\right)$ & $\tilde{p}_{s} \cdot \sin ^{2}\left(\pi \frac{v}{v_{f}}\right) \cdot\left(\cos \left(\pi \frac{2 v}{v_{f}}\right)+i \cos \left(\pi \frac{2 v}{v_{f}}+\delta\right)\right)$ \\
\hline
\end{tabular}

Next, we examined the simplified versions of the problem in question, namely the non-phantom $\left(\xi_{1}=\xi_{2}=1\right)$ collapses of neutral and electrically charged scalar fields leading to Schwarzschild and Reissner-Nordström spacetimes, respectively. The results are consistent with those published, e.g., in Refs. [16, 19, 20].

The dynamical Schwarzschild spacetime corresponding to the outcome of the collapse of a neutral scalar field [16] in fact results from the evolution of dilaton field under the influence of gravitation ( $E D$-collapse). Its structure for $\tilde{p}_{k}=0.1$ is shown in Fig 1 a. There is one apparent horizon visible in the spacetime, namely $r_{, v}=0$. For $v \rightarrow \infty$ it settles down to an event horizon, which is situated along $u=1.06$. It surrounds the central spacelike singularity located along the line $r=0$, which becomes singular at $u=1.38$ (the peak on the diagram).

The dynamical Reissner-Nordström spacetime emerging from the collapse of an electrically charged scalar field (EMevolution) 20 for $\tilde{p}_{s}=0.6$ is shown in Fig $1 \mathrm{~b}$. There are two horizons in the spacetime. The outer apparent horizon, $r_{, v}=0$, after the dynamical part of evolution, when $v \rightarrow \infty$, coincides with an event horizon located along $u=0.84$. The inner horizon, namely the Cauchy horizon is located at future null infinity, i.e., at $v \rightarrow \infty$. The line $r=0$ becomes central spacelike singularity at $u=3.04$.

\section{RESULTS}

As was mentioned in the introduction, the main aim of our research was to investigate the results of the dynamical collapse of an electrically charged scalar field in the presence of dilaton field, while one or both types of fields are phantom. All the possible configurations were presented in Table [1. Because of the fact that the structure of spacetimes emerging from the dynamical collapse depends on the unknown coupling constant $\alpha$ [29], we take into account $\alpha=-1$ and $\alpha=0$. These values are particularly interesting because they refer to analytical models discussed in the literature. Specifically, $\alpha=-1$ refers to the low-energy string theory, while when $\alpha$ vanishes the dynamical collapse of an electrically charged scalar field in presence of an uncoupled dilaton field takes place. On the contrary, the electric coupling constant does not exert any influence on the evolution, so we assume that it is equal to $e=0.5$. The results of the numerical computations are depicted on Penrose diagrams, i.e., we plot lines of constant $r(u, v)$ in the $(v, u)$-plane.

The types of the initial field profiles, which were used in our numerical computations are presented in Table II The $\left(f_{D}\right)$-family refers to dilaton field, while the family $\left(f_{S}\right)$ is connected with electrically charged scalar field. The free family parameter $\tilde{p}$ denotes an amplitude of the particular field. Namely, $\tilde{p}_{s}$ is responsible for the electrically charged scalar field, while $\tilde{p}_{k}$ for the dilaton field.

At the beginning we will consider the separate evolutions of an electrically charged scalar field with phantom coupling of Maxwell field to gravity ( $E \bar{M}$-collapse) and phantom dilaton field ( $E \bar{D}$-collapse). Then we shall pay attention to the evolutions when more than one field is involved (phantom-EMD-collapses). In these situations the one-parameter cases will be considered at first. It means that the amplitudes of both collapsing fields will be taken to be equal and their common value will be denoted by $\tilde{p}$. Afterwards, in order to complete the physical picture of the results of the examined process, for each of phantom-EMD evolutions we shall discuss the behavior of the system in the case when the amplitudes of the considered fields differ. To be precise, we describe the changes in the spacetime structures appearing when one of the fields has a constant amplitude and the amplitude of the other field varies. The constant amplitudes of the electrically charged scalar field and dilaton field were chosen to be equal to $\tilde{p}_{s}=0.6$ and $\tilde{p}_{k}=0.1$, respectively. Such choice was motivated by the fact that these evolutions in the nonphantom case lead to the dynamical Reissner-Nordström and Schwarzschild spacetimes as was described above. At the same time in the phantom cases, as will be explained in sections IVA and IVB, they lead to Schwarzschild and non-singular spacetimes, which are typical for particular phantom evolutions. In view of the bewildering complexity of the considered dynamical collapse it will enable us to generalize about the role of the accompanying field (which 
amplitude varies) in the phenomena under consideration.

\section{A. Einstein-phantom Maxwell collapse}

To begin with, we shall consider the first of the simplified versions of the theory under consideration. Namely, we will discuss spacetime structures emerging in the process of the dynamical collapse of the charged scalar field in the case of Einstein-phantom Maxwell theory ( $E \bar{M}$-evolution). The representative structure is shown in Fig 2 , The parameter characterizing the initial amplitude of the considered field was chosen as $\tilde{p}_{s}=0.6$. It turned out that the most striking feature of the resulting spacetime is the fact that despite the presence of an electric charge only one apparent horizon is observed. The outcome of the considered dynamical collapse is similar to the one in the case of Schwarzschild spacetime, which results from the evolution of a self-gravitating electrically neutral scalar field (Fig 1 a). The single apparent horizon $r_{, v}=0$ surrounds the spacelike central singularity and settles along an event horizon for $v \rightarrow \infty$.

The event horizon is a null hypersurface situated along $u=0.78$. In comparison with the case of the dynamical collapse of the electrically charged scalar field in non-phantom Einstein-Maxwell case (EM-evolution), observed for the same parameter $\tilde{p}_{s}=0.6$ and depicted in Fig $1 \mathrm{~b}$, the emerging event horizon is situated along the smaller value of retarded time. As far as the point, where the line $r=0$ becomes singular is concerned (peak on the Penrose diagram), one can draw the same conclusion. Namely, in phantom dynamical collapse it corresponds to $u=2.98$, whereas in non-phantom Einstein-Maxwell evolution the singularity arises slightly later as was described above.

To conclude, one can observe that in the case of Einstein-phantom Maxwell dynamical collapse the structure of the issuing spacetime is Schwarzschild-like and the black hole forms earlier in terms of the retarded time comparing to the non-phantom Einstein-Maxwell evolution. The comparison between $E \bar{M}$ and $E M$ cases allows us to draw an interesting conclusion concerning the correlation between the attractive/repulsive nature of forces and spacetime structures. As was already mentioned, gravitational force is always attractive and supports the collapse. The electrostatic force in EM-evolution is repulsive and the Reissner-Nordström spacetime is the outcome of some kind of a compromise between the two forces. In contrast, the electrostatic force in $E \bar{M}$-evolution is attractive just as gravitational force. In this case a simpler, Schwarzschild-like spacetime emerges.

\section{B. Einstein-phantom dilaton collapse}

To proceed further, let us consider the second simplification, i.e., dynamical collapse in the case of Einstein-phantom dilaton system ( $E \bar{D}$-evolution), where dilaton field evolves under the influence of gravitational field. This case simply refers to the evolution of phantom electrically neutral scalar field. A vast range of the parameters $\tilde{p}_{k}$ characterizing the initial amplitude of the field was examined. The results of the numerical calculations for $\tilde{p}_{k}$ equal to $0.25,0.5$ and 1 are shown in Fig3. An important notion concerning the evolution in question is the fact that for every considered dilaton field amplitude the emerging spacetime is non-singular, that is black holes do not form. The only influence of the parameter $\tilde{p}_{k}$ on the resulting spacetime structure is the fact that the bigger value of $\tilde{p}_{k}$ we take the more significantly $r$-coordinate varies in the vicinity of the center of spacetime (pushing out the lines of $r=$ const. in the vicinity of the point $u=v=0$ ). The aforementioned behaviour is in complete contrast to the dynamical evolution in Einstein-scalar theory (ED-evolution). In this case the dynamical collapse of non-phantom scalar field, for appropriately large values of the parameter $\tilde{p}_{k}$ results in a singular, Schwarzschild-type spacetime, as was shown in Fig 1 a.

Summing it all up, the influence of the phantom nature of the scalar field is even more extreme in the electrically neutral case than in the charged one. In Einstein-phantom dilaton theory black holes do not appear in comparison with Einstein-scalar case, where Schwarzschild-type black holes are obtained. It may be also explained in terms of the nature of acting forces. Attractive gravitational force cooperates with an attractive force during $E D$-collapse and competes with repulsive dilatonic force in $E \bar{D}$-evolution. It turns out that in the latter case gravity is not able to overcome the repulsive nature of phantom field.

\section{Einstein-phantom Maxwell-dilaton collapse}

The first theory concerning the collapse of both considered fields, namely the dilaton and electrically charged scalar ones, is Einstein-phantom Maxwell-dilaton theory ( $E \bar{M} D$-evolution). It should be emphasized that there are no differences between spacetime structures resulting from the process for the coupling constant $\alpha$ equal to -1 and 0 while values of field amplitudes do not differ. In Fig 4 the outcome of an evolution in $E \bar{M} D$-theory for both considered values of the dilatonic coupling constant is presented. The value of parameter $\tilde{p}$ was taken to be equal to 0.06 and 
0.175. For small values of $\tilde{p}$ the spacetime is non-singular. For its bigger values the Schwarzschild-like black hole forms. We achieve a single apparent horizon $r_{, v}=0$ surrounding spacelike singularity. When $v$-coordinate tends to infinity the apparent horizon coincides with the event horizon of a newly born black hole.

This situation is also in agreement with the previous analyzes of the nature of acting forces. In this case all of them are attractive, hence for the sufficiently large value of the amplitudes the simplest singular, Schwarzschild-type structure forms.

In order to proceed to considerations of how various fields influence on the dynamical collapse in question, we kept an amplitude of electrically charged scalar field $\tilde{p}_{s}$ constant and equal to 0.6 and varied the amplitude of dilaton field. The structures of spacetimes for $\alpha=-1,0$ and $\tilde{p}_{k}$ equal to 0.01 and 0.09 are shown in Fig 5 . Then, we performed computations the other way round. Namely, we changed an amplitude of electrically charged scalar field $\tilde{p}_{s}$ while keeping the other amplitude $\tilde{p}_{k}$ constant and equal to 0.1. In Fig 6 we depicted spacetime structures for the amplitude of electrically charged scalar field equal to 0.35 and 0.6 for both considered values of dilatonic coupling constant. In all the cases we obtained dynamic Schwarzschild spacetimes.

For small values of a varying amplitude we observed Schwarzschild-like spacetimes like the one shown in Fig $1 \mathrm{p}$, that is a central spacelike singularity surrounded by one apparent horizon $r_{v}=0$, which after a dynamical part of evolution settles along an event horizon for $v \rightarrow \infty$. For larger values of a varying amplitude slightly different behaviour was revealed, namely two dynamical stages during the evolution were observed. After achieving a constant value of $u$-coordinate an apparent horizon $r_{, v}=0$ changes its position to another value of retarded time, which is smaller than the previous one. As $v \rightarrow \infty$ the horizon again settles along $u=$ const. indicating the position of an event horizon of a final black hole. Of course a spacelike singularity is situated in the center of it. Such structure also corresponds to Schwarzschild-type.

An increase of $\tilde{p}_{k}$ results in earlier (in terms of retarded time) appearance of a point, where the line $r=0$ becomes singular (Fig 5). This increase is also connected with the fact that an event horizon is situated along a smaller constant value of $u$-coordinate. The decrease of a value of retarded time corresponding to a location of an event horizon is more significant than the decrease of $u$ referring to the first singular point at $r=0$. Within the set of solutions with altering amplitude of phantom counterpart of electrically charged scalar field (Fig, 6), the changes of $\tilde{p}_{s}$ do not affect the location of a point, where the line $r=0$ becomes singular. At the same time the location of the event horizon moves towards lower values of $u$-coordinate when $\tilde{p}_{s}$ increases.

The energy momentum tensor $(9)-(12)$ for $E \bar{M} D$-theory in the case of $\alpha=-1$ has the following form:

$$
\begin{aligned}
T_{\mu \nu}(F, \phi) & =-e^{-2 \phi} T_{\mu \nu}(F)+T_{\mu \nu}(\phi), \\
T_{\mu \nu}\left(\phi, F, \psi, \psi^{*}, A\right) & =\tilde{T}_{\mu \nu}\left(\psi, \psi^{*}, A\right)+T_{\mu \nu}(F, \phi),
\end{aligned}
$$

while for $\alpha=0$ :

$$
\begin{aligned}
T_{\mu \nu}(F, \phi) & =-T_{\mu \nu}(F)+T_{\mu \nu}(\phi), \\
T_{\mu \nu}\left(\phi, F, \psi, \psi^{*}, A\right) & =e^{2 \phi} \tilde{T}_{\mu \nu}\left(\psi, \psi^{*}, A\right)+T_{\mu \nu}(F, \phi),
\end{aligned}
$$

where $\tilde{T}_{\mu \nu}\left(\psi, \psi^{*}, A\right), T_{\mu \nu}(F)$ and $T_{\mu \nu}(\phi)$ denote the parts connected with electrically charged, Maxwell and dilaton fields, respectively. The coexisting effects of the dilaton field and phantom counterpart of an electrically charged scalar field result in an appearance of Schwarzschild black hole. Even though the changes of the amplitudes of particular fields change their relative impact on the collapse, its outcome is in all cases similar.

It is worth mentioning that analytic studies of singular static configurations in $E \bar{M} D$-theory were conducted in Refs. [7, 9]. It turned out that in the considered case the solutions are Schwarzschild-type, i.e., the spacetimes consist of one horizon surrounding central singularity. This conclusion was confirmed by our computations also in the case of dynamical collapse.

\section{Einstein-Maxwell-phantom dilaton collapse}

The next type of the theory we shall be interested in is Einstein-Maxwell-phantom dilaton gravity ( $E M \bar{D}$-evolution). In this theory the electrically charged scalar field coupled to phantom dilaton field collapses gravitationally. The plots bounded with the dynamical collapse in $E M \bar{D}$-evolution, for the dilatonic coupling constant equal to -1 and 0 are shown in Figs 7 and 8 , respectively. In our considerations, for the case of $\alpha=-1$, we have chosen the parameter $\tilde{p}$ to be equal to $0.5,0.85$ and 1.1. When the coupling constant $\alpha$ is equal to zero $\tilde{p}$ equals to $0.1,0.8$ and 1.1.

For all values of the family parameter $\tilde{p}$ the non-singular spacetimes emerge for both considered values of $\alpha$. For small values of the parameter $\tilde{p}$ there are no horizons in spacetime. Refining our numerical studies to the larger values of $\tilde{p}$, we obtain an apparent horizon $r_{, u}=0$ for small values of retarded time. For $\alpha=-1$, it settles along constant 
$u$-coordinate for $v \rightarrow \infty$, while for $\alpha=0$ it forms an open loop with ending points at $v \rightarrow \infty$. It happens that for even bigger values of the amplitudes another type of an apparent horizon, namely $r_{, v}=0$, emerges. We observe both $r_{, u}=0$ and $r_{, v}=0$ types of the apparent horizons. However, their presence in spacetime is not connected with the appearance of the singularity.

For $\alpha=-1$ the results are depicted in Fig,7. One observes that the appearance of $r_{, u}=0$ and $r_{, v}=0$ apparent horizons for small values of $u$-coordinate develops a gap between $r=$ const. lines at $v \rightarrow \infty$. The adjacent lines move away very quickly. As was mentioned above, for large values of parameter $\tilde{p}$ both types of apparent horizons emerge. For the coupling constant $\alpha=0$ the results are shown in Fig.8. One gets that for large values of the parameter $\tilde{p}$ and small values of the $u$-coordinate as well as for large values of both retarded and advanced times, both $r_{, u}=0$ and $r_{, v}=0$ apparent horizons exist in the non-singular spacetime.

In the considered $E M \bar{D}$-theory the repulsive electromagnetic and dilatonic forces compete with the attractive gravitational force after all leading to non-singular spacetimes with single or multiple horizons.

In order to get a deeper insight into the physical processes taking place during $E M \bar{D}$-collapse we shall vary amplitudes of phantom dilaton and of electrically charged scalar field. We commence with altering an amplitude of phantom dilaton while an amplitude of the other field remains constant and equal to $\tilde{p}_{s}=0.6$. For $\alpha=-1$ the structures formed when $\tilde{p}_{k}$ equals to $0.15,0.2$ and 0.25 are shown in Fig.9. For small values of the parameter $\tilde{p}_{k}$ we get the singular spacetime with two kinds of apparent horizons, but when the amplitude of phantom dilaton grows the spacetime becomes non-singular. The growing amplitude of phantom dilaton field suppresses emergence of a singularity. This result seems to be intuitive because phantom field contributes negatively to the total energy of the system.

As was mentioned above, for small values of the phantom dilaton amplitude the spacetime became singular but with two types apparent horizons $r_{, u}=0$ and $r_{, v}=0$ in the domain of integration. For values of amplitudes not exceeding 0.15 there are two branches of $r_{, v}=0$ horizon. The first one settles along an event horizon for $v \rightarrow \infty$. With an increasing value of the phantom dilaton field amplitude, the singularity as well as the horizon appears at increasing $u$-coordinate. The second branch of the horizon $r_{, v}=0$ lies inside the first one and it begins at the point, where the line of singular $r=0$ ends. The apparent horizon $r_{, u}=0$ is situated inside the second branch of $r_{, v}=0$ horizon and begins at the same point. For small values of parameter $\tilde{p}_{k}$ these two lines almost coincide. The structure of an area beyond the horizon $r_{, u}=0$ is impossible to interpret fully and clearly, because calculations cannot be carried out until $r=0$ and they end at some positive value of $r$. Such structure with two branches of $r, v=0$ apparent horizon and an apparent horizon $r_{, u}=0$ inside them may be interpreted as a dynamical wormhole [24]. On the other hand, for greater values of the parameter $\tilde{p}_{k}$ (larger than 0.2 ) the two branches of the horizon $r, v=0$ form a loop with two ends coinciding with ending points of singular part of line $r=0$. The horizon $r, u=0$ remains outside it and continuously tends towards smaller values of $u$-coordinate as $v \rightarrow \infty$. This structure corresponds to an unphysical situation of naked singularity in spacetime 24. For even bigger values of amplitudes of dilaton field, from approximately $\tilde{p}_{k}=0.25$, the spacetime is non-singular with no visible horizons.

A close inspection of the energy momentum tensor $\sqrt{9}-12$ for $E M \bar{D}$-theory in the considered case of $\alpha=-1$ reveals that one gets the following:

$$
\begin{aligned}
T_{\mu \nu}(F, \phi) & =e^{-2 \phi} T_{\mu \nu}(F)-T_{\mu \nu}(\phi), \\
T_{\mu \nu}\left(\phi, F, \psi, \psi^{*}, A\right) & =\tilde{T}_{\mu \nu}\left(\psi, \psi^{*}, A\right)+T_{\mu \nu}(F, \phi) .
\end{aligned}
$$

It can be seen that the factor $e^{-2 \phi}$ diminishes the influence of the Maxwell field on the collapse, i.e., the bigger amplitude of phantom dilaton field we consider the smaller influence is of the $U(1)$-gauge field we obtain. When $\tilde{p}_{k}$ increases the strong repulsive effect of the phantom dilaton field begins to play the dominant role leading to non-singular spacetimes.

Different situation takes place for Einstein-Maxwell-phantom dilaton collapse without dilaton coupling, that is for $\alpha=0$. The structures of the emerging spacetimes for $\tilde{p}_{k}$ equal to 0.05 and 0.8 are shown in Fig 10 . The spacetimes are non-singular for all values of the parameter $\tilde{p}_{k}$. It means that the presence of phantom dilaton field, not interacting with the rest of matter in spacetime, prevents black hole formation. The spacetime is non-singular with both types of horizons at first and then, for $\tilde{p}_{k}$ exceeding 0.7 , only one open loop-shaped apparent horizon $r, u=0$ is observable for the small values of the $u$-coordinate.

On the other hand, the energy momentum tensor for $\alpha=0$ is provided by

$$
\begin{aligned}
T_{\mu \nu}(F, \phi) & =T_{\mu \nu}(F)-T_{\mu \nu}(\phi), \\
T_{\mu \nu}\left(\phi, F, \psi, \psi^{*}, A\right) & =e^{2 \phi} \tilde{T}_{\mu \nu}\left(\psi, \psi^{*}, A\right)+T_{\mu \nu}(F, \phi) .
\end{aligned}
$$

Eq. 59 reveals that the growth of the phantom dilaton field amplitude plays the dominant role while the factor $e^{2 \phi}$ also enlarges the influence of the electrically charged scalar field. Therefore due to these facts the domination of the phantom dilaton field seems to be a key factor in the process of the dynamical collapse for this case. 
The next set of solutions referring to Einstein-Maxwell-phantom dilaton theory involves spacetimes emerging from a collapse when an amplitude of phantom dilaton field is constant and an amplitude of electrically charged scalar field varies. The value of the constant amplitude was set as $\tilde{p}_{k}=0.1$. In Fig 11 the structures of spacetimes emerging from the collapse for $\alpha=-1$ were depicted. The amplitudes of the scalar field $\tilde{p}_{s}$ were chosen to be equal to 0.3 and 0.6 , respectively. One can observe that for small values of the electrically charged scalar field amplitude the repulsive character of phantom dilaton field combined with small amount of the electrically charged scalar field results in the nonsingularity of the emerging spacetime. On the other hand, when the role of phantom dilaton field is diminished, i.e., the amplitude of electrically charged scalar field grows, one attains the singular spacetime with two branches of $r_{, v}=0$ apparent horizon - the outer and the inner one as well as an apparent horizon $r_{, u}=0$ located within them. One remarks that a dynamical wormhole structure appears. Namely, the emerging black hole gains phantom dilaton matter violating the null energy condition and this phenomenon causes formation of a wormhole.

As may be inferred from Eq. (58) the growth of an electrically charged scalar field amplitude is able to neutralize the diminishing effect of the factor $e^{-2 \phi}$ coexisting with the repulsive tendency of phantom dilaton field. It finally leads to the formation of a dynamic wormhole.

The structures of spacetimes emerging from $E M \bar{D}$-evolution with constant value of an amplitude $\tilde{p}_{k}$ and dilatonic coupling constant $\alpha=0$ are shown in Fig,12. The amplitude of charged scalar field $\tilde{p}_{s}$ was set as equal to 0.2 and 0.7 . The spacetimes for all values of a varying amplitude are non-singular. For small values of it there are no horizons visible in the domain of integration. In turn, for bigger values of $\tilde{p}_{s}$ apparent horizons $r_{, v}=0$ and $r_{, u}=0$ appear in spacetime, but none of them reach the line $r=0$, which for this reason does not become singular. The emergence of the apparent horizons is accompanied by squeezing the lines of $r=$ const. in the vicinity of the line $r=0$, in the area lying above the $r_{, v}=0$ horizon in terms of the $u$-coordinate.

From Eq. (59) we can observe that the factor containing phantom dilaton field $e^{2 \phi}$ plays an important role together with the growth of the electrically charged scalar field amplitude in the domain of integration. This is closely related to the fact that phantom dilaton field suppresses appearance of black hole, while an increasing amplitude of the electrically charged scalar field relatively to dilaton field amplitude forces the formation of horizons in the underlying spacetime.

\section{E. Einstein-phantom Maxwell-phantom dilaton collapse}

The last examined case is Einstein-phantom Maxwell-phantom dilaton theory (E$\overline{M D}$-evolution). The resulting spacetimes formed during the process of the dynamical collapse are depicted in Figs 13 and 14 . The plots were made for the dilaton coupling constant equal to -1 and 0 , respectively. For $\alpha=-1$ the amplitudes of the considered fields were chosen to be equal to $0.3,0.4$ and 0.5 . In the case of $\alpha=0$, the parameter $\tilde{p}$ was equal to 0.2 and 0.5 .

For both values of $\alpha$ the spacetimes for small values of $\tilde{p}$ are non-singular without any horizons. Similarly to the previously analyzed dynamical collapse, in the majority of cases both types of apparent horizons, namely $r, v=0$ and $r_{, u}=0$ are visible in the domain of integration. Moreover, the appearance of the apparent horizon $r_{, v}=0$ for larger values of the free family parameter always ensues from the formation of singularity in the considered spacetime. What is more, the bigger value of the parameter one considers the earlier in terms of retarded time the singularity at $r=0$ appears.

In Fig 13 , plotted for the coupling constant $\alpha=-1$, one observes a loop-shaped part of the apparent horizon $r_{, v}=0$ near the peak of the line $r=0$, where it becomes singular. The size of a loop-shaped apparent horizon decreases with increasing field parameter $\tilde{p}$. What is more, the loop surrounds the singular part of the $r=0$ line. Outside the loop the singularity is absent or impossible to localize. When $v$-coordinate tends to infinity, the apparent horizon $r, v=0$ settles along constant $u$-coordinate. Moreover, the other apparent horizon begins at the point, where the singular $r=0$ ends and tends towards smaller values of retarded time as $v \rightarrow \infty$. As was already stated in the previous section, such structure corresponds to naked singularity in spacetime.

In Fig 14 we presented the results for the uncoupled case, that is for $\alpha=0$. One notices that for the large values of parameter $\tilde{p}$ the spacetime relaxes to the one containing naked singularity. There is a loop-shaped apparent horizon surrounding the central spacelike singularity and it tends towards $u=$ const. as $v \rightarrow \infty$. Additionally, there are two branches of the horizon $r_{, u}=0$. One of them is connected with the naked singularity. The other one forms an open loop similar to the ones observed in spacetimes emerging from the $E M \bar{D}$-evolutions conducted for $\alpha=0$.

In conclusion it may be stated that spacetimes emerging from the collapse in Einstein-phantom Maxwell-phantom dilaton theory with equal amplitudes of both fields are either non-singular or they contain naked singularities. In this case only the dilatonic force is repulsive, the other two forces have attractive character.

Let us turn our attention to the evolutions with a constant amplitude of phantom electrically charged scalar field equal to $\tilde{p}_{s}=0.6$. For $\alpha=-1$ we considered the values of phantom dilaton field amplitude $\tilde{p}_{k}$ equal to 0.1 and 0.3. The spacetimes emerging from these evolutions are presented in Fig. 15 . For all values of $\tilde{p}_{k}$ the structure of the 
spacetime resembles Schwarzschild one, i.e., there is an apparent horizon $r_{, v}=0$ coinciding with an event horizon at $v \rightarrow \infty$ and surrounding central spacelike singularity. The peak, where the line $r=0$ becomes singular practically does not change its $u$-location as $\tilde{p}_{k}$ increases. On the contrary, the value of $u$-coordinate corresponding to the location of the event horizon increases while the amplitude of phantom dilaton field becomes bigger.

In the considered case of $\alpha=-1$ the energy momentum tensor $(9-12$ for $E \overline{M D}$-theory is given by:

$$
\begin{aligned}
T_{\mu \nu}(F, \phi) & =-e^{-2 \phi} T_{\mu \nu}(F)-T_{\mu \nu}(\phi), \\
T_{\mu \nu}\left(\phi, F, \psi, \psi^{*}, A\right) & =\tilde{T}_{\mu \nu}\left(\psi, \psi^{*}, A\right)+T_{\mu \nu}(F, \phi) .
\end{aligned}
$$

Contrary to the case described by relations (58), where we observe dynamic wormhole spacetimes, now the phantom coupling of Maxwell field causes that the outcome is similar to the Schwarzschild spacetime. It should be emphasized that it happens despite of the influence of phantom dilaton field, which previously prevented black hole formation due to its repulsive nature.

On the other hand, Fig,16 illustrates spacetime structures resulting from a collapse in Einstein-phantom Maxwellphantom dilaton theory without dilaton coupling, i.e., for $\alpha=0$. The values of phantom dilaton field were set as equal to 0.05 and 0.2 . For small values of $\tilde{p}_{k}$ the spacetime is singular with one apparent horizon $r_{, v}=0$ surrounding singularity visible in the domain of integration. Nearby the point, where the line $r=0$ becomes singular the apparent horizon almost coincides with the singularity. At some point it moves away from the line $r=0$ towards slightly smaller values of $v$-coordinate and then settles along constant $u$ for $v \rightarrow \infty$. Since it becomes null hypersurface there we may predict that its location at large values of advanced time indicates the location of the event horizon. Although the structure in the vicinity of the point, where the line $r=0$ becomes singular (beyond the event horizon) is different from the one presented in Fig.1 1 , we conclude that it may be interpreted as Schwarzschild-like. The emerging object consists of a central spacelike singularity surrounded by one apparent horizon coinciding with an event horizon at $v \rightarrow \infty$. The length of a part, where the apparent horizon is very close to $r=0$ decreases with an increase of an amplitude of phantom dilaton field. The location of the point, where the line $r=0$ becomes singular slightly decreases in terms of $u$ while the varying amplitude increases. For larger values of a varying amplitude, naked singularity and an additional open loop-shaped branch of an apparent horizon $r_{, u}=0$ appear in the spacetime.

Consider now the energy momentum tensor for the studied $\alpha=0$ case. It implies

$$
\begin{aligned}
T_{\mu \nu}(F, \phi) & =-T_{\mu \nu}(F)-T_{\mu \nu}(\phi), \\
T_{\mu \nu}\left(\phi, F, \psi, \psi^{*}, A\right) & =e^{2 \phi} \tilde{T}_{\mu \nu}\left(\psi, \psi^{*}, A\right)+T_{\mu \nu}(F, \phi) .
\end{aligned}
$$

Having in mind Eq. (59), the case when no formation of a black hole was indicated, now we observe that phantom coupling of Maxwell field provides the singularity in the emerging spacetime and the formation of a Schwarzschild-like black hole or naked singulatrity. Similarly to the case of $\alpha=-1$ black hole formation takes place in spite of the presence of the repulsive phantom dilaton field.

The next set of solutions corresponding to $E \overline{M D}$-theory refers to the computations conducted for a constant phantom dilaton field amplitude $\tilde{p}_{k}=0.1$ and altering amplitude of phantom counterpart of an electrically charged scalar field. The spacetimes ensuing from the collapse for dilatonic coupling constant $\alpha=-1$ and values of an electrically charged scalar field $\tilde{p}_{s}$ equal to 0.25 and 0.5 are shown in Fig.17. For small values of an amplitude $\tilde{p}_{s}$ the spacetime is non-singular, while for larger values it becomes Schwarzschild-type. There is one apparent horizon $r_{, v}=0$ indicating the location of an event horizon for $v \rightarrow \infty$. It surrounds central spacelike singularity like in Fig.17. The damping effect of $e^{-2 \phi}$ in $(60)$ is dominant at first and the phantom dilaton field plays the most important role. But with the passage of the increase of the phantom counterpart of the electrically charged scalar field amplitude the situation reverses and this field manages to force black hole formation. Just one can conclude that instead of the diminishing factor $e^{-2 \phi}$, the sign of energy momentum tensor of $U(1)$-gauge field combined with the increasing value of the amplitude of the electrically charged scalar field are of the crucial importance for the formation of Schwarzschild-like black hole.

The diagrams of spacetimes for $\alpha=0$ are illustrated in Fig, 18 . The amplitude of the phantom electrically charged scalar field was equaled to 0.35 and 0.65 . For small values of $\tilde{p}_{s}$ the spacetime is non-singular. The structures obtained when the amplitude $\tilde{p}_{s}$ increases are Schwarzschild-like with an additional branch of an $r_{, u}=0$ apparent horizon at small values of the retarded time.

On the other hand, a close inspection of (61) reveals that together with the dominating factor $e^{2 \phi}$, the opposite sign of the Maxwell term again occurs decisive for the singularity to appear in the domain under inspection.

As was noticed in the above subsection, the very familiar situation as described in $E M \bar{D}$-theory, i.e., black hole solution with effectively one event horizon in the case of $\alpha^{2} \rightarrow 1$ [7, takes place in $E \overline{M D}$-gravity. This analytical result obtained in the static case was confirmed in our simulations of the dynamical collapse. 


\section{CONCLUSIONS}

In our paper we studied the problem of the dynamical collapse of charged scalar field in the Einstein-Maxwell-dilaton theory with phantom coupling of the adequate fields. These fields are allowed to violate the null energy condition. The landscape of static and spherically symmetric solutions for the system under consideration abounds in eleven different not only asymptotically flat spacetimes related to sixteen various causal structures 9 . Such a rich collection of solutions aroused our interest especially in the context of the dynamical evolution.

At first, it was revealed that in Einstein-phantom Maxwell evolution the structure of the resulting spacetime resembles Schwarzschild-like spacetime despite of the presence of the electrically charged $U(1)$-gauge field. In this case phantom nature of the electrically charged scalar field forces Schwarzschild-like structure formation instead of Reissner-Nordström-like in the non-phantom case. What is more, the resulting event horizon in the case of $E \bar{M}$ dynamical collapse emerges slightly earlier, in terms of the retarded time, than in the case of the dynamical collapse in EM-theory. Such structure remains in agreement with the one described in non-dynamical case [7, 9].

Then, taking into account Einstein-phantom dilaton theory, one observes that for every considered amplitude of the dilaton field the resulting spacetime is non-singular. There is no formation of a black hole. Such behaviour is in complete contrast to the dynamical collapse in Einstein-dilaton (Einstein-scalar) evolution, which leads to the formation of Schwarzchild-like spacetime [16. In this case the phantom nature of scalar field prevents a singularity as well as event horizons formation due to the repulsive character of the field.

On its turn, in Einstein-phantom Maxwell-dilaton case there are no qualitative differences among the structures of the emerging spacetimes for the different values of the coupling constant $\alpha$. One arrives at the Schwarzschild-like black hole formation, with a single horizon surrounding spacelike singularity. Dilaton field and phantom counterpart of electrically charged scalar field cooperate in Schwarzschild-type structure formation. These dynamically obtained structures only partially confirm the static solutions for $E \bar{M} D$-theory obtained in 9 . From a wide variety of possibilities during the dynamical evolution the simplest one with only one horizon surrounding spacelike singularity is preferred.

As far as Einstein-Maxwell-phantom dilaton theory is concerned, the obtained structures are far more complicated than in the previous cases. First of all, two types of apparent horizons are observed in the spacetime, namely lines of $r_{, u}=0$ and $r_{, v}=0$. Moreover, the appearance of an apparent horizon is not necessarily connected with the emergence of the singularity. For the dilatonic coupling constant equal to -1 dynamical wormhole spacetimes are observed in case when an amplitude of phantom dilaton field is considerably smaller than an amplitude of an electrically charged scalar field. In fact one has a formation of the black hole which gains phantom dilaton field which violates null energy condition. This phenomenon provides a formation of the wormhole structure. For $\alpha=0$ the spacetimes are non-singular. It means that an uncoupled phantom dilaton field prevents black hole formation due to its repulsive character. It seems that in the case of $E M \bar{D}$-collapse the diversity of solutions manifests itself most clearly. Obviously, as was noticed in [9, 10, multiple horizons separating distinct areas are observed. Moreover, as was anticipated in static cases [7, 9, wormhole structures in the case of phantom scalar field and non-phantom vector field were obtained also during the dynamical collapse. Interestingly, our computations also provided an evidence of the existence of the dynamical interconversion between black holes and wormholes, which results from the dynamics of the phantom field in the considered spacetime [31.

Finally we note that, in the case of Einstein-phantom Maxwell-phantom dilaton theory, also both types of the apparent horizons are visible in the domain of integration. The obtained structures correspond to the non-singular or Schwarzschild-type spacetimes or they contain naked singularities. Naked singularities mainly appear when the amplitudes of both collapsing fields are equal. On the other hand, the dynamical collapse results in Schwarzschild-like spacetime when an amplitude of the dilaton field is considerably smaller than the other one. Hence it may be stated that the considered $E \overline{M D}$-evolution again leads to the simplest structure with a singularity surrounded by an event horizon among those presented in [9].

During our numerical simulations of a dynamical collapse in phantom-EMD system we obtained an extensive collection of the spacetime structures. They are summarized in Table III together with the structures emerging from the collapse without phantom coupling. An analysis of energy-momentum tensors and the nature of forces acting in the considered system combined with the study of the emerging spacetime structures, led us to the general conclusions concerning the role of particular fields and the dilatonic coupling during the collapse in question.

It turned out that the non-phantom dilaton field supports the formation of Schwarzschild-type singular spacetimes. On the other hand, its phantom counterpart suppresses black hole formation due to its strongly repulsive nature. It also facilitates the formation of multiple horizons not necessarily connected with the singularity. The non-phantom Maxwell field seems to be less repulsive than phantom dilaton field and thus it favors Reissner-Nordström-type spacetime as an outcome of the dynamical collapse. What is more, phantom Maxwell field, similarly to the dilaton field, supports the creation of the simplest singular, Schwarzschild-like structure despite its phantom coupling to gravity.

Considering the role of a complex scalar field during the collapse, it seems to act just as a carrier of the Maxwell field. 
TABLE III: Spacetime structures emerging from the phantom gravitational collapse. The overlining indicates phantom field. The structures of spacetimes resulting from the evolutions in non-phantom gravity are summarized at the bottom for comparison. Symbols: $\mathcal{A}$ - attractive, $\mathcal{R}$ - repulsive, $n s$ - non-singular spacetime, $S$ - Schwarzschild-type spacetime, $R N$ - ReissnerNordström-type spacetime, $W H$ - wormhole, $N S$ - naked singularity. Superscripts: ${ }^{*}$ - additional horizons in spacetime, ${ }^{(2)}-$ two-stage collapse. Arrows: $\uparrow-$ an increase of a particular amplitude, $\rightarrow-$ the direction of changes while the amplitude varies.

\begin{tabular}{|c|c|c|c|c|c|c|c|}
\hline \multirow{2}{*}{ Evolution } & \multicolumn{2}{|c|}{ Forces } & \multirow{2}{*}{$\begin{array}{c}\text { Dilatonic } \\
\text { coupling constant }\end{array}$} & \multirow{2}{*}{$\begin{array}{c}\text { Energy-momentum } \\
\text { tensor }\end{array}$} & \multirow{2}{*}{$\tilde{p}_{s}=\tilde{p}_{k} \uparrow$} & \multirow{2}{*}{$\begin{array}{c}\tilde{p}_{s}=\text { const. } \\
\tilde{p}_{k} \uparrow\end{array}$} & \multirow{2}{*}{$\begin{array}{c}\tilde{p}_{k}=\text { const. } \\
\tilde{p}_{s} \uparrow\end{array}$} \\
\hline & $\overline{M / \bar{M}}$ & $D / \bar{D}$ & & & & & \\
\hline$E \bar{M}$ & $\mathcal{A}$ & - & - & $\tilde{T}_{\mu \nu}(\psi)-T_{\mu \nu}(F)$ & - & - & $n s \rightarrow S$ \\
\hline$E \bar{D}$ & - & $\mathcal{R}$ & - & $-T_{\mu \nu}(\phi)$ & - & $n s$ & - \\
\hline$E \bar{M} D$ & $\mathcal{A}$ & $\mathcal{A}$ & $\begin{array}{c}\alpha=-1 \\
\alpha=0\end{array}$ & $\begin{array}{c}\tilde{T}_{\mu \nu}(\psi)-e^{-2 \phi} T_{\mu \nu}(F)+T_{\mu \nu}(\phi) \\
e^{2 \phi} \tilde{T}_{\mu \nu}(\psi)-T_{\mu \nu}(F)+T_{\mu \nu}(\phi)\end{array}$ & $n s \rightarrow S$ & $S \rightarrow S^{(2)}$ & $S \rightarrow S^{(2)}$ \\
\hline$E M \bar{D}$ & $\mathcal{R}$ & $\mathcal{R}$ & $\begin{array}{c}\alpha=-1 \\
\alpha=0\end{array}$ & $\begin{array}{c}\tilde{T}_{\mu \nu}(\psi)+e^{-2 \phi} T_{\mu \nu}(F)-T_{\mu \nu}(\phi) \\
e^{2 \phi} \tilde{T}_{\mu \nu}(\psi)+T_{\mu \nu}(F)-T_{\mu \nu}(\phi)\end{array}$ & $\begin{array}{l}n s \rightarrow n s^{*} \\
n s \rightarrow n s^{*}\end{array}$ & $\begin{array}{c}W H \rightarrow N S \rightarrow n s \\
n s^{*}\end{array}$ & $\begin{array}{l}n s \rightarrow W H \\
n s \rightarrow n s^{*}\end{array}$ \\
\hline$E \overline{M D}$ & $\mathcal{A}$ & $\mathcal{R}$ & $\begin{array}{c}\alpha=-1 \\
\alpha=0\end{array}$ & $\begin{array}{c}\tilde{T}_{\mu \nu}(\psi)-e^{-2 \phi} T_{\mu \nu}(F)-T_{\mu \nu}(\phi) \\
e^{2 \phi} \tilde{T}_{\mu \nu}(\psi)-T_{\mu \nu}(F)-T_{\mu \nu}(\phi)\end{array}$ & $\begin{array}{c}n s \rightarrow N S \\
n s \rightarrow N S^{*}\end{array}$ & $\begin{array}{l}S \\
S \rightarrow N S^{*}\end{array}$ & $\begin{array}{c}n s \rightarrow S \\
n s \rightarrow S^{*}\end{array}$ \\
\hline$E M 20$ & $\mathcal{R}$ & - & - & $\tilde{T}_{\mu \nu}(\psi)+T_{\mu \nu}(F)$ & - & - & $n s \rightarrow R N$ \\
\hline$E D$ [16] & - & $\mathcal{A}$ & - & $T_{\mu \nu}(\phi)$ & - & $n s \rightarrow S$ & - \\
\hline$E M D[29]$ & $\mathcal{R}$ & $\mathcal{A}$ & $\begin{array}{c}\alpha=-1 \\
\alpha=0\end{array}$ & $\begin{array}{c}\tilde{T}_{\mu \nu}(\psi)+e^{-2 \phi} T_{\mu \nu}(F)+T_{\mu \nu}(\phi) \\
e^{2 \phi} \tilde{T}_{\mu \nu}(\psi)+T_{\mu \nu}(F)+T_{\mu \nu}(\phi)\end{array}$ & $\begin{array}{c}n s \rightarrow S \\
n s \rightarrow R N\end{array}$ & $\begin{aligned} S & \rightarrow S^{(2)} \\
R N & \rightarrow R N^{(2)}\end{aligned}$ & $\begin{aligned} S & \rightarrow S^{(2)} \\
R N & \rightarrow R N^{(2)}\end{aligned}$ \\
\hline
\end{tabular}

It does not influence the obtained structures distinctly. Such situation is in agreement with the previously examined simpler case of the collapse of electrically charged scalar field in Einstein gravity [20].

Considering the dilatonic coupling it may be revealed that $\alpha=-1$ diminishes the influence of Maxwell field on the evolution and thus enhances the role of the dilaton field in its course. The dilatonic coupling constant equal to zero acts the other way round, i.e., it reduces the role of dilaton field during the collapse and indirectly enlarges the meaning of Maxwell field by acting on the complex electrically charged scalar field.

\section{Acknowledgments}

AN was supported by Human Capital Programme of European Social Fund sponsored by European Union. MR was partially supported by the grant of the National Science Center 2011/01/B/ST2/00408.

[1] N.Arkani-Hamed, H.Ch.Cheng, M.A.Luty, and S.Mukoyama, JHEP 0405, 251 (2004).

[2] S.Permutter, et al., Astrophys. J. 483, 565 (1997),

B.P.Schmidt, et al., ibid. 507, 46 (1998),

A.G.Riess, A.J. 116, 1009 (1998).

[3] C.L.Bennett et al., Astrophys. J. Suppl. 148, 1 (2003).

[4] D.J.Eisenstein et al. Astrophys. J. 633, 560 (2005).

[5] R.R.Caldwell, Phys. Lett. B 545, 23 (2002),

M.Dąbrowski, T.Stachowiak and M.Szydłowski, Phys. Rev. D 68, 103519 (2003). 
[6] H.G.Ellis, J. Math. Phys. 14, 104 (1973), E.I.Novikova and I.D.Novikov, Phys. Rev. D 81, 104034 (2010).

[7] G.W.Gibbons and D.A.Rasheed, Nucl. Phys. B 476, 515 (1996).

[8] G.Clement and J.C.Fabris, Class. Quantum Grav. 16, 323 (1999).

[9] G.Clement, J.C.Fabris, and M.E.Rodrigues, Phys. Rev. D 79, 064021 (2009).

[10] M.Azreg-Ainou, G.Clement, J.C.Fabris, and M.E.Rodrigues, Phys. Rev. D 83, 124001 (2011).

[11] M.Heusler, Black Holes Uniqueness Theorems (Cambridge University Press, Cambridge, England, 1996), K.S.Thorne, Black Holes and Time Warps (W.W.Norton and Company, New York, 1994).

[12] W.A.Hiscock, Phys. Lett. A 83, 110 (1981).

[13] A.Ori, Phys. Rev. Lett. 67, 789 (1991), A.Ori, Phys. Rev. Lett. 68, 2117 (1992), L.M.Burko and A.Ori, Phys. Rev. Lett. 74, 1064 (1995), A.Ori and D.Gorbonos, J. Math. Phys. 48, 092502 (2007), D.Gorbonos and G.Wolansky, J. Math. Phys. 48, 092503 (2007).

[14] E.Poisson and W.Israel, Phys. Rev. D 41, 1796 (1990), E.Poisson and W.Israel, Phys. Rev. Lett. 63, 1663 (1989).

[15] M.L.Gnedin and N.Y.Gnedin, Class. Quantum Grav. 10, 1083 (1993).

[16] R.S.Hamade and J.M.Stewart, Class. Quantum Grav. 13, 497 (1996).

[17] P.R.Brady and J.D.Smith, Phys. Rev. Lett. 75, 1256 (1995).

[18] S.Ayal and T.Piran, Phys. Rev. D 56, 4768 (1997).

[19] S.Hod and T.Piran, Phys. Rev. Lett. 81, 1554 (1998), S.Hod and T.Piran, Gen. Rel. Grav. 30, 1555 (1998).

[20] Y.Oren and T.Piran, Phys. Rev. D 68, 044013 (2003).

[21] E.Sorkin and T.Piran, Phys. Rev. D 63, 084006 (2001).

[22] E.Sorkin and T.Piran, Phys. Rev. D 63, 124024 (2001).

[23] S.E.Hong, D.Hwang, E.D.Stewart, and D.Yeom, Class. Quantum Grav. 27, 045014 (2010).

[24] D.Hwang and D.Yeom, Phys. Rev. D 84, 064020 (2011).

[25] D.Hwang and D.Yeom, Class. Quantum Grav. 27, 205002 (2010).

[26] J.A.Gonzáles and F.S.Guzmán, Phys. Rev. D 79, 121501(R) (2009).

[27] A.Doroshkevich, J.Hansen, D.Novikov, I.Novikov, D.Park, and A.Shatskiy, Phys. Rev. D 81, 124011 (2010).

[28] A.K.M.Masood-ul-Alam, Class. Quantum Grav. 14, 2649 (1993),

M.Rogatko, Class. Quantum Grav. 14, 2425 (1997), M.Rogatko, ibid. 19, 875 (2002),

M.Mars and W.Simon, Adv. Theor. Math. Phys. 6, 279 (2003), M.Rogatko, Phys. Rev. D 58, 044011 (1998), M.Rogatko, ibid. 59, 104010 (1999), M.Rogatko, ibid. 82, 044017 (2010), S.Yazadijev, ibid. 82, 124050 (2010).

[29] A.Borkowska, M.Rogatko and R.Moderski, Phys. Rev. D 83, 084007 (2011).

[30] D.Christodoulou, Comm. Pure Appl. Math. 46, 1131 (1993).

[31] S.A.Hayward, Phys. Rev. D 79, 124001 (2009). 
(a)

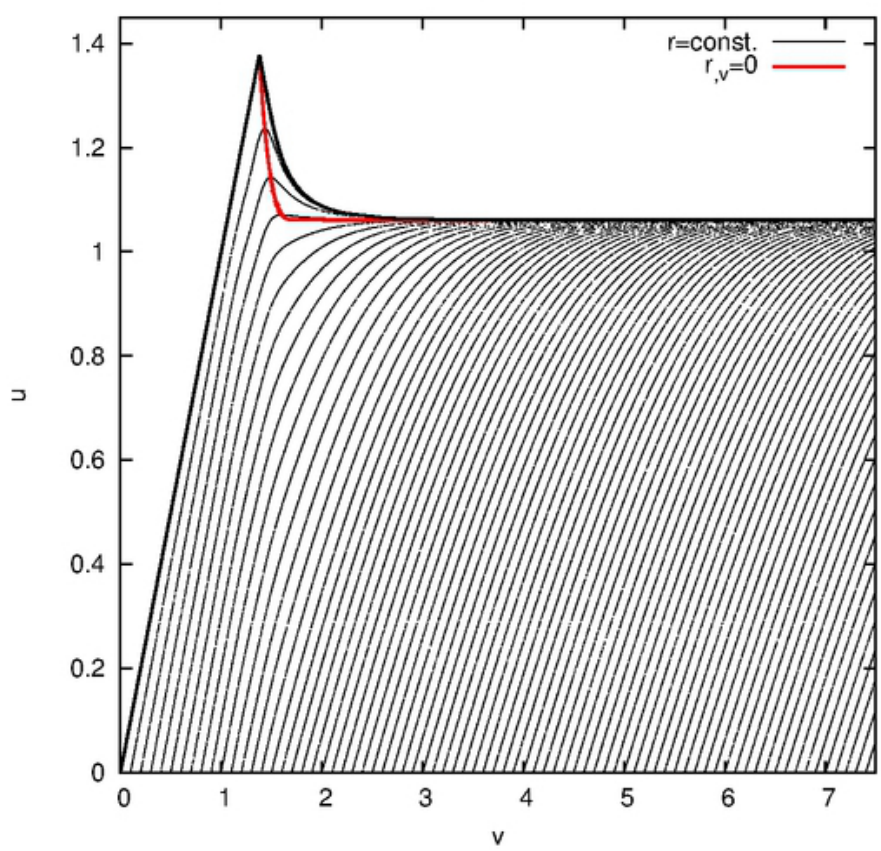

(b)

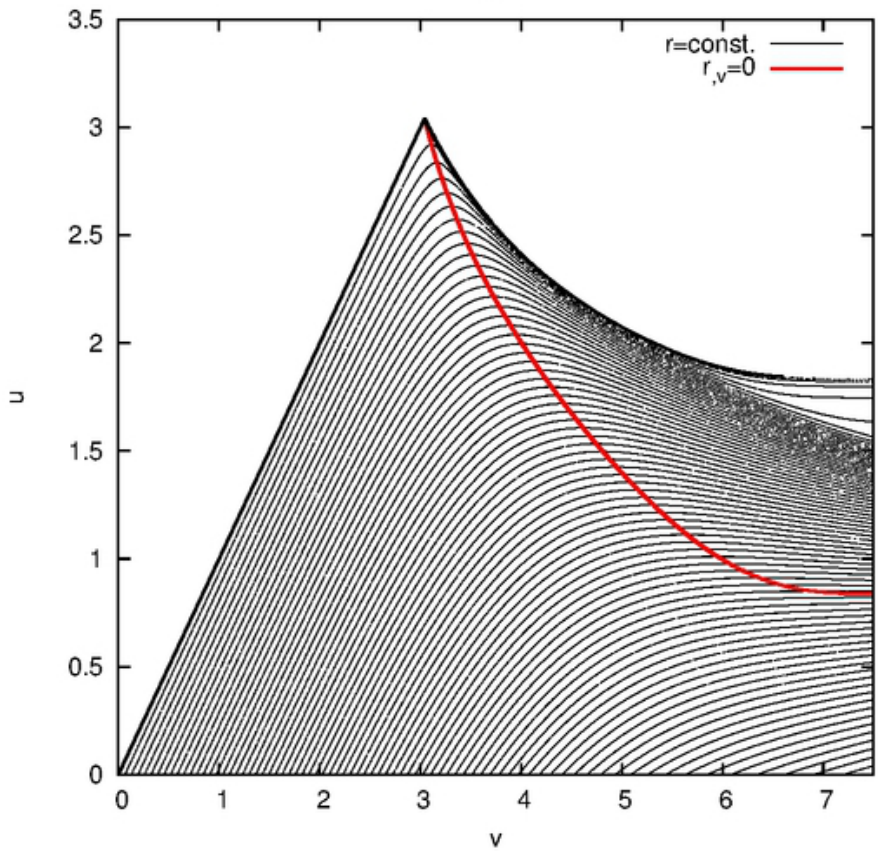

FIG. 1: (color online). Penrose diagrams of the dynamical (a) Schwarzschild and (b) Reissner-Nordström spacetimes obtained for the field amplitudes equal to $\tilde{p}_{k}=0.1$ and $\tilde{p}_{s}=0.6$, respectively. 


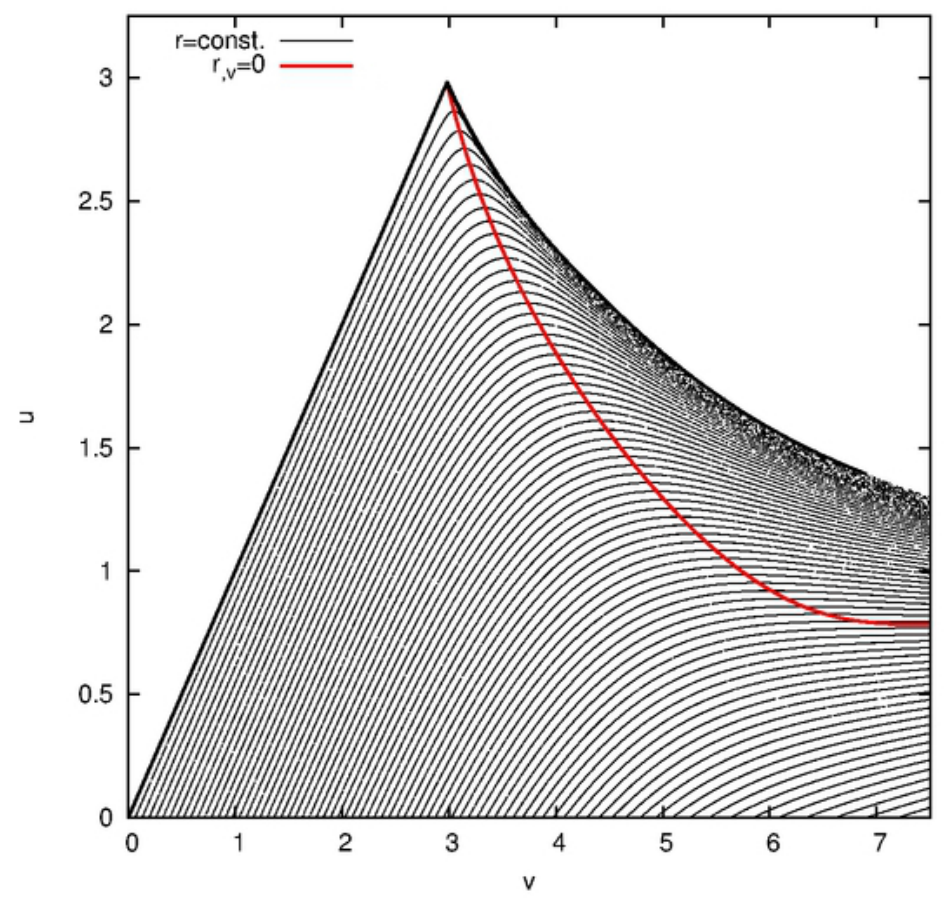

FIG. 2: (color online). Lines of constant $r$ in the $(v u)$-plane for $E \bar{M}$-evolution with the electric coupling constant $e=0.5$. The family parameter is set to be $\tilde{p}_{s}=0.6$. 
(a)

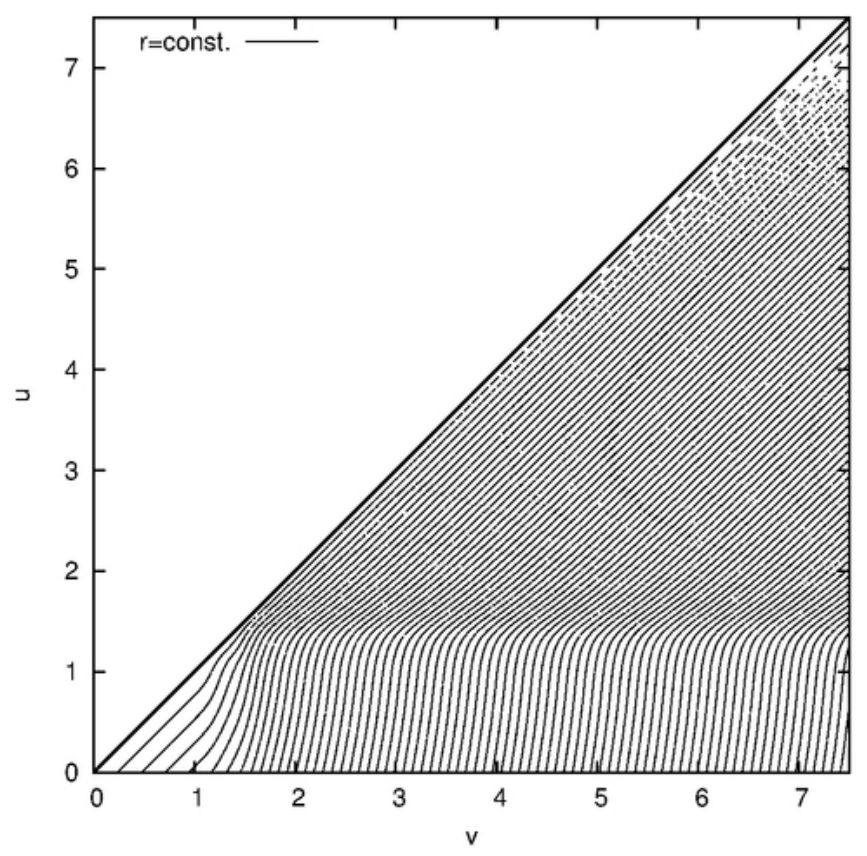

(b)

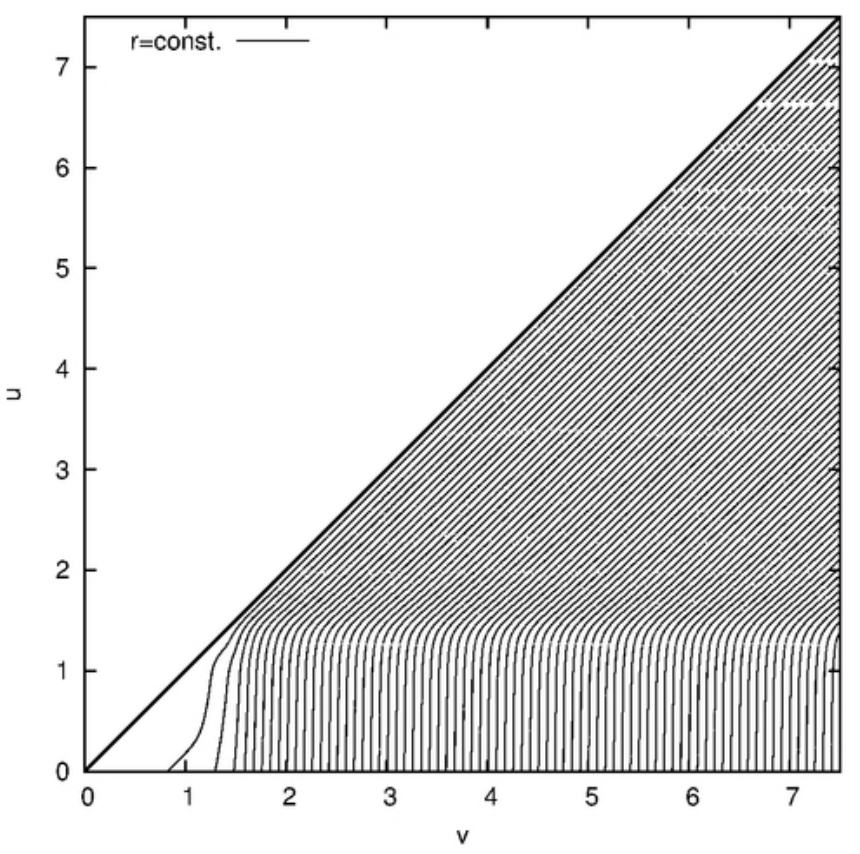

(c)

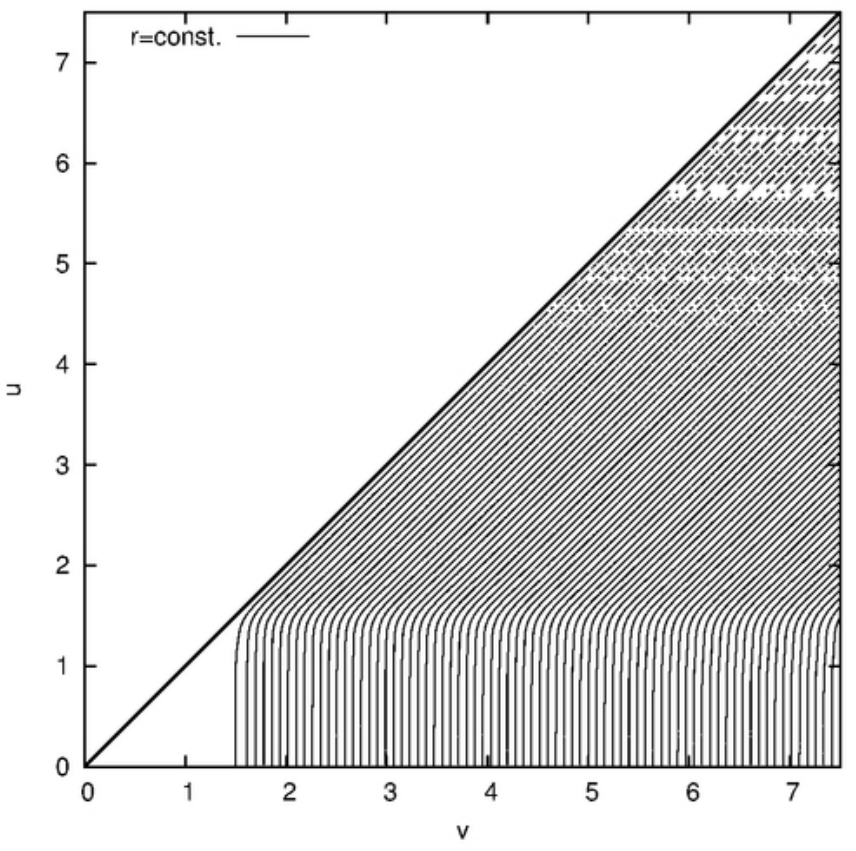

FIG. 3: (color online). Lines of constant $r$ in the $(v u)$-plane for $E \bar{D}$-evolution with the family parameter equal to (a) $\tilde{p}_{k}=0.25$, (b) $\tilde{p}_{k}=0.5$ and (c) $\tilde{p}_{k}=1$. 
(a)

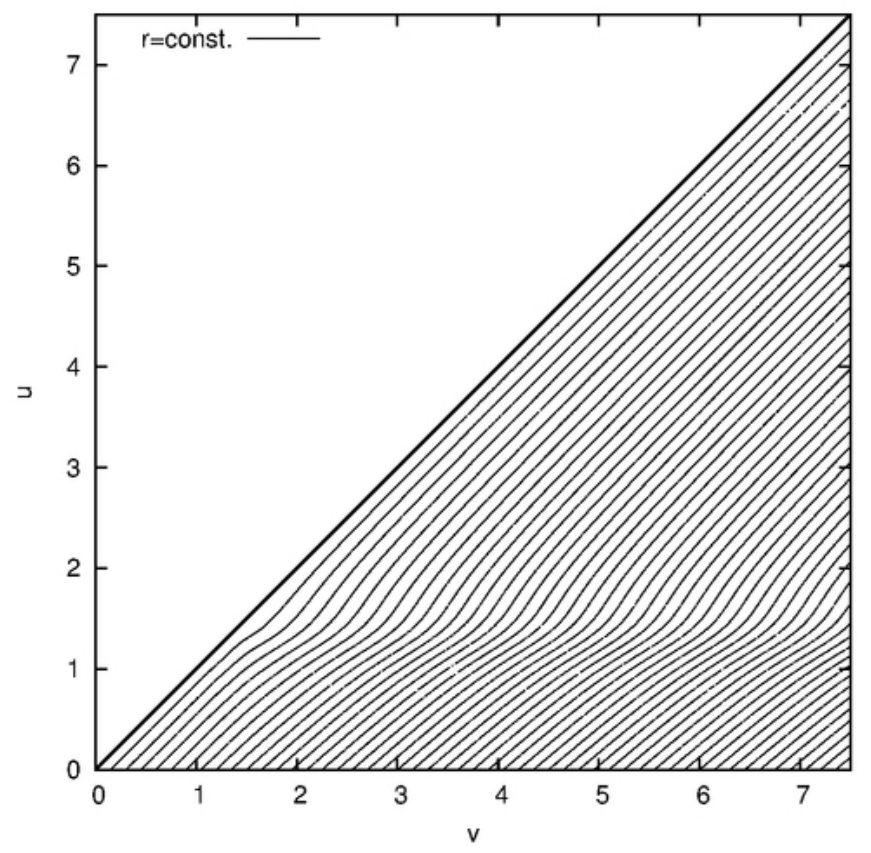

(b)

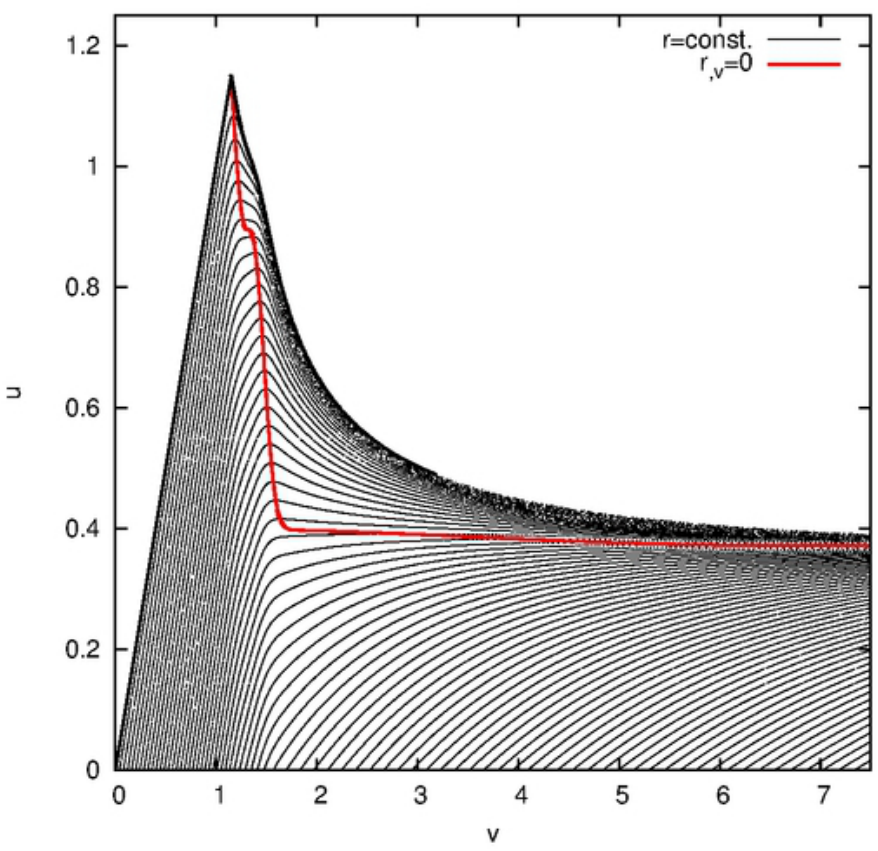

FIG. 4: (color online). Lines of constant $r$ in the the $(v u)$-plane for $E \bar{M} D$-evolution with the electric coupling constant $e=0.5$ and the dilatonic coupling constant $\alpha$ equal to -1 and 0 . The family parameter is taken to be (a) $\tilde{p}=0.06$ and (b) $\tilde{p}=0.175$. 
(a)

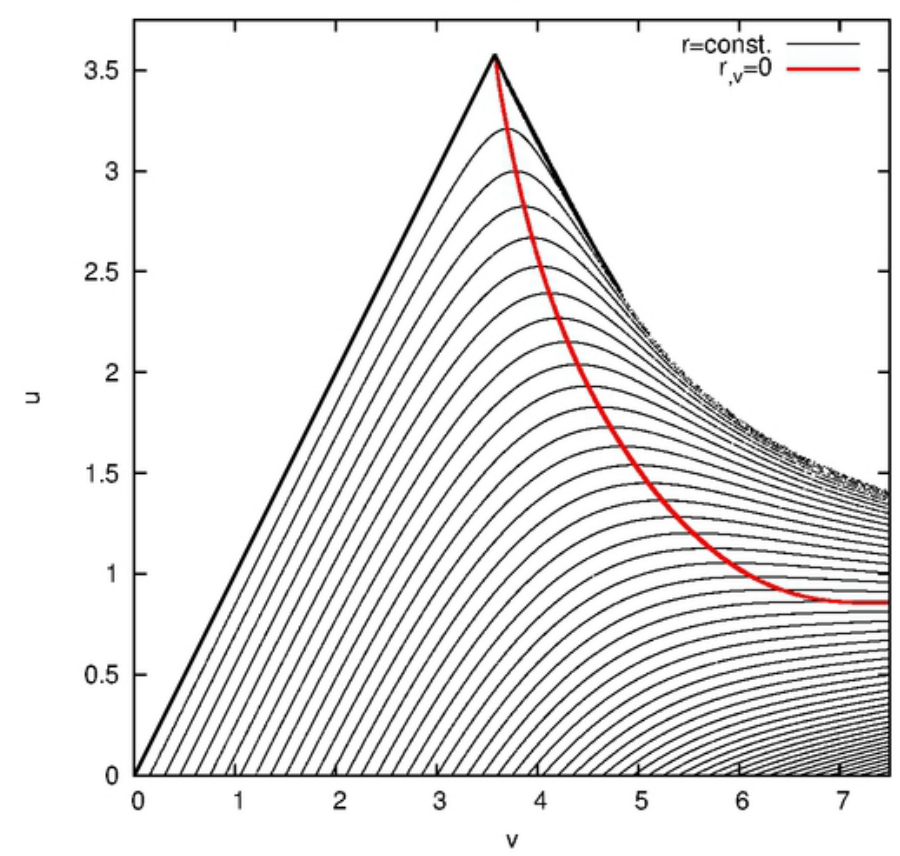

(b)

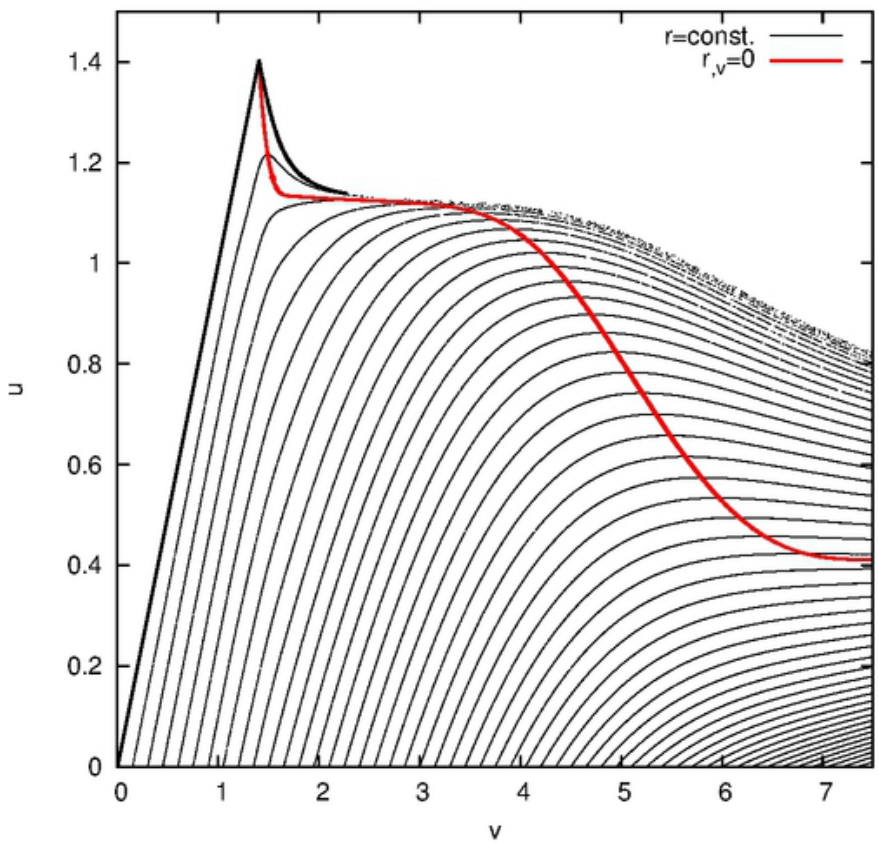

FIG. 5: (color online). Lines of constant $r$ in $(v u)$-plane for the $E \bar{M} D$ evolution with a varying dilaton field amplitude. The coupling constants have the same values as in Fig 4 The family parameter for the electrically charged scalar field $\tilde{p}_{s}=0.6$, while for the dilaton field (a) $\tilde{p}_{k}=0.01$ and (b) $\tilde{p}_{k}=0.09$. 
(a)

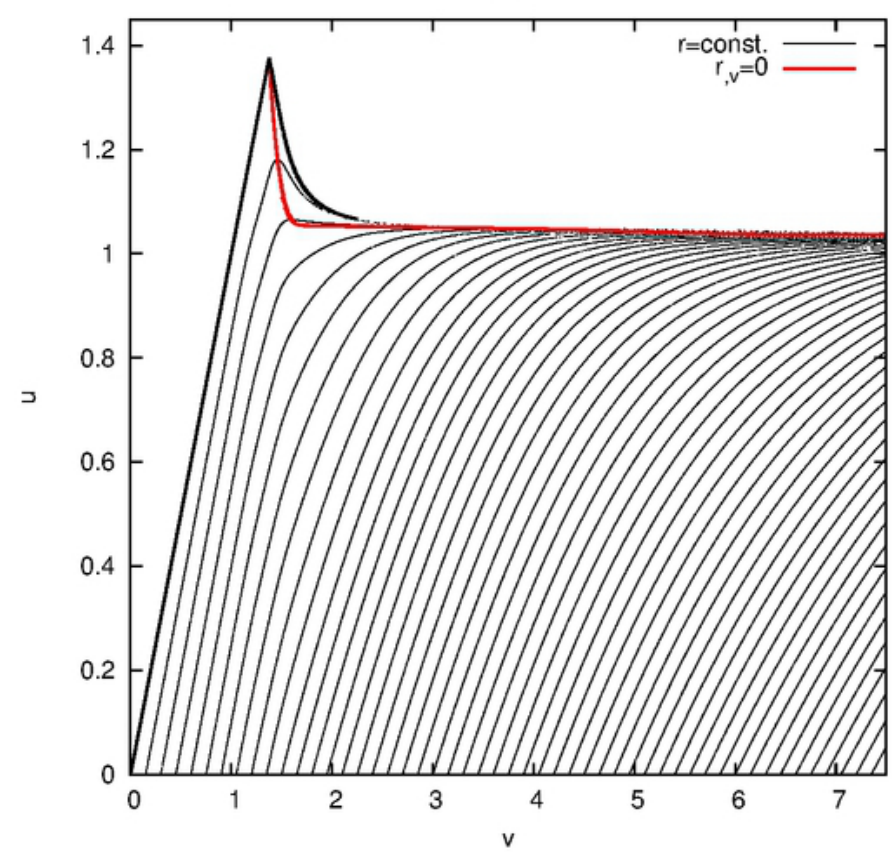

(b)

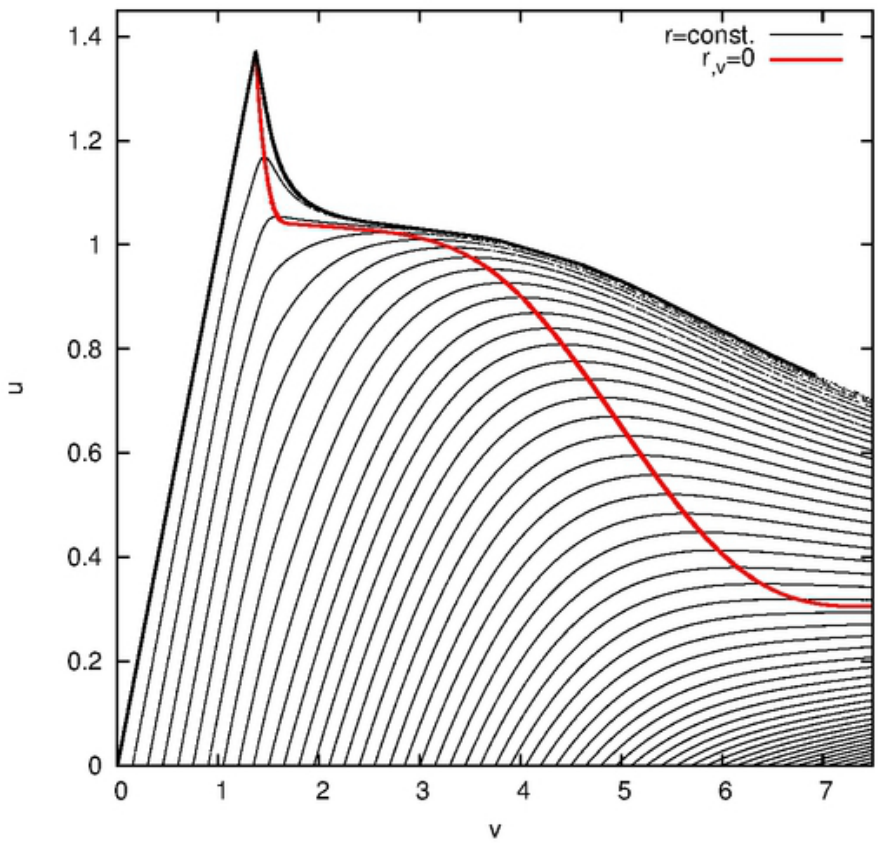

FIG. 6: (color online). Lines of constant $r$ in the (vu)-plane for the $E \bar{M} D$ evolution with varying electrically charged scalar field amplitude. The coupling constants have the same values as in Fig 4 The family parameter for the dilaton field is equal to $\tilde{p}_{k}=0.1$, while for the electrically charged scalar field field is set to be (a) $\tilde{p}_{s}=0.35$ and (b) $\tilde{p}_{s}=0.6$, respectively. 
(a)

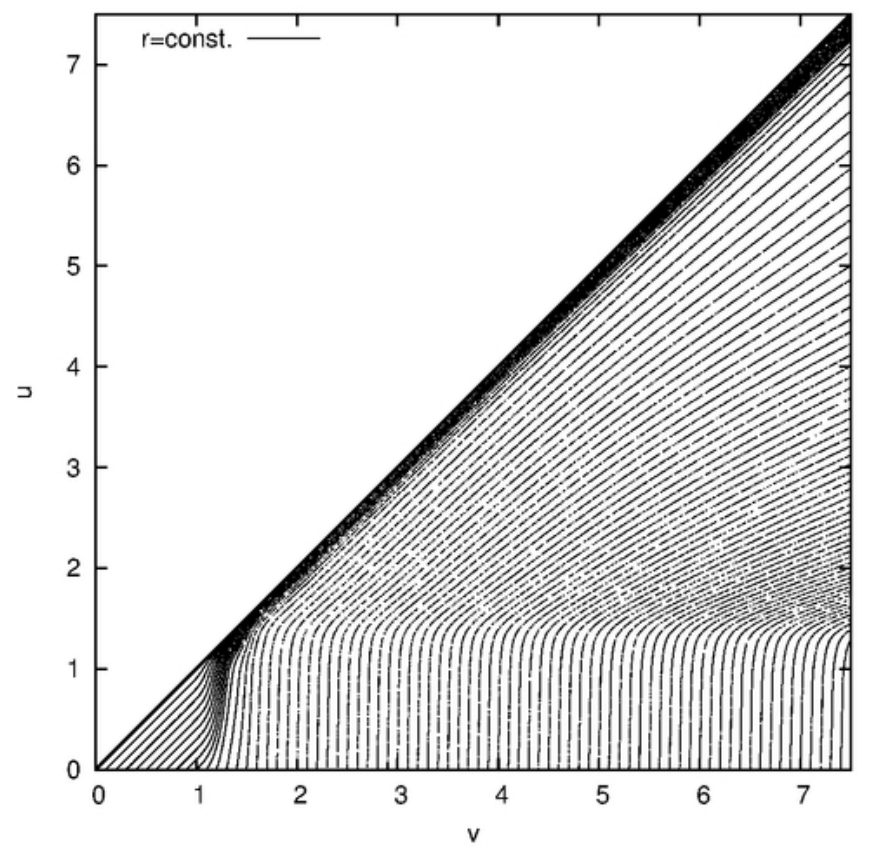

(b)

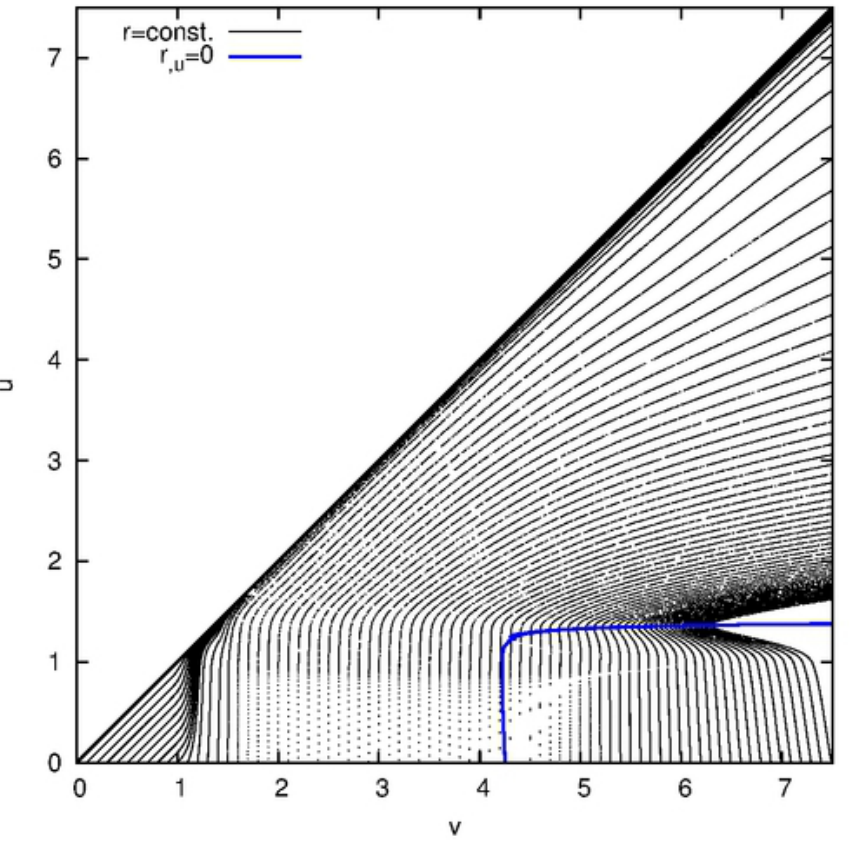

(c)

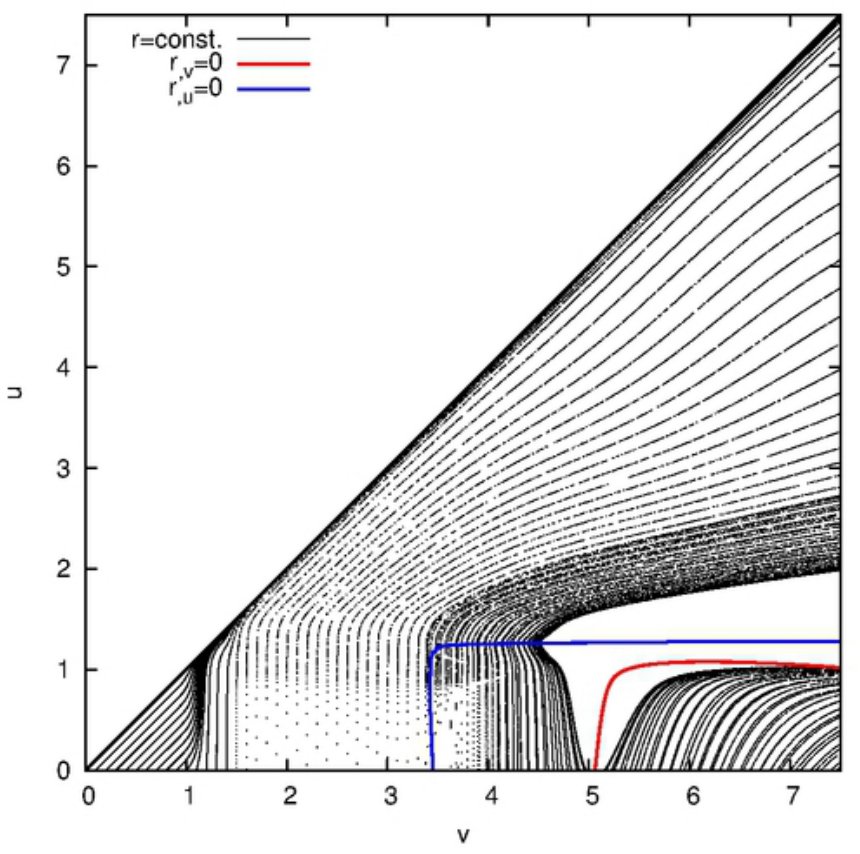

FIG. 7: (color online). Lines of constant $r$ in the $(v u)$-plane for $E M \bar{D}$-evolution with the electric and the dilatonic coupling constants equal to $e=0.5$ and $\alpha=-1$, respectively. The family parameter is equal to (a) $\tilde{p}=0.5$, (b) $\tilde{p}=0.85$ and (c) $\tilde{p}=1.1$. 
(a)

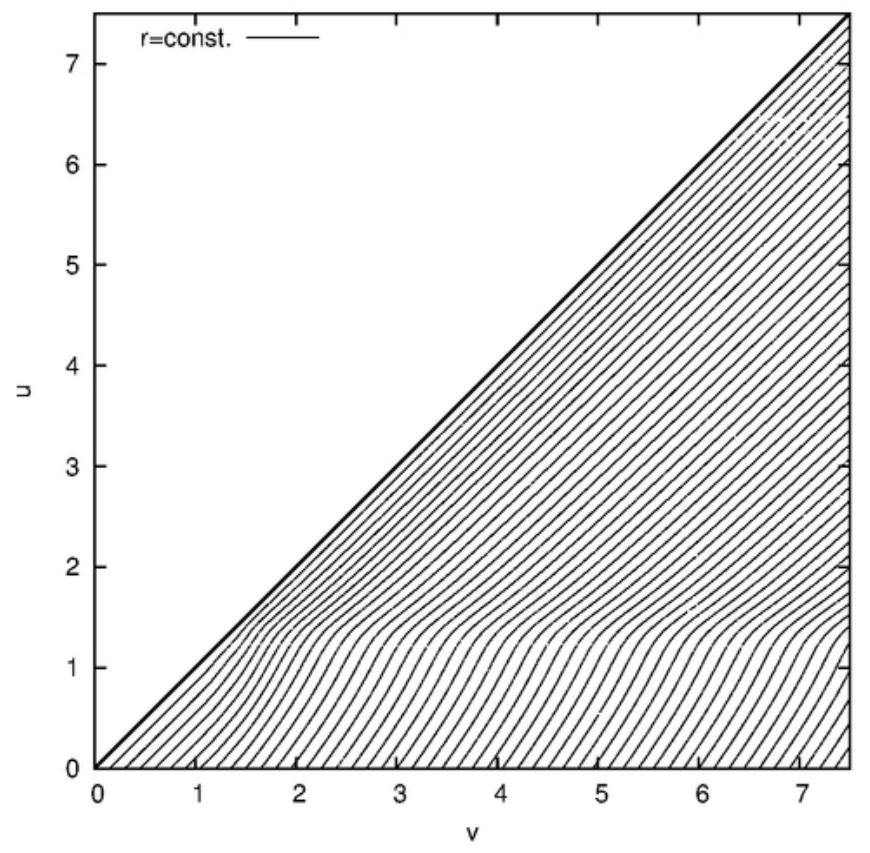

(b)

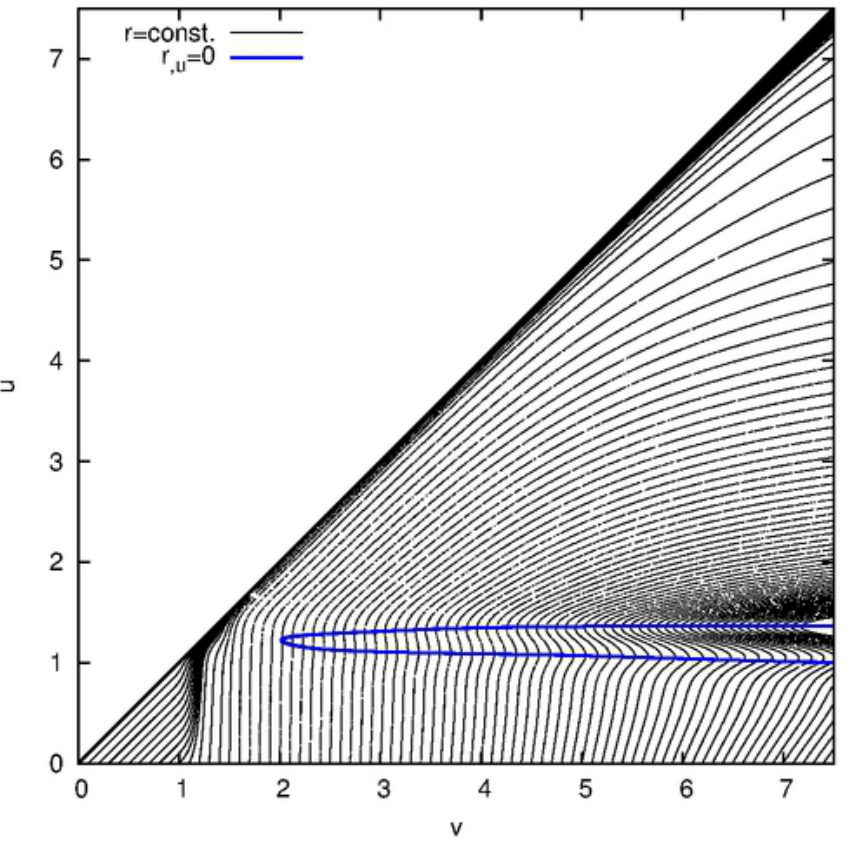

(c)

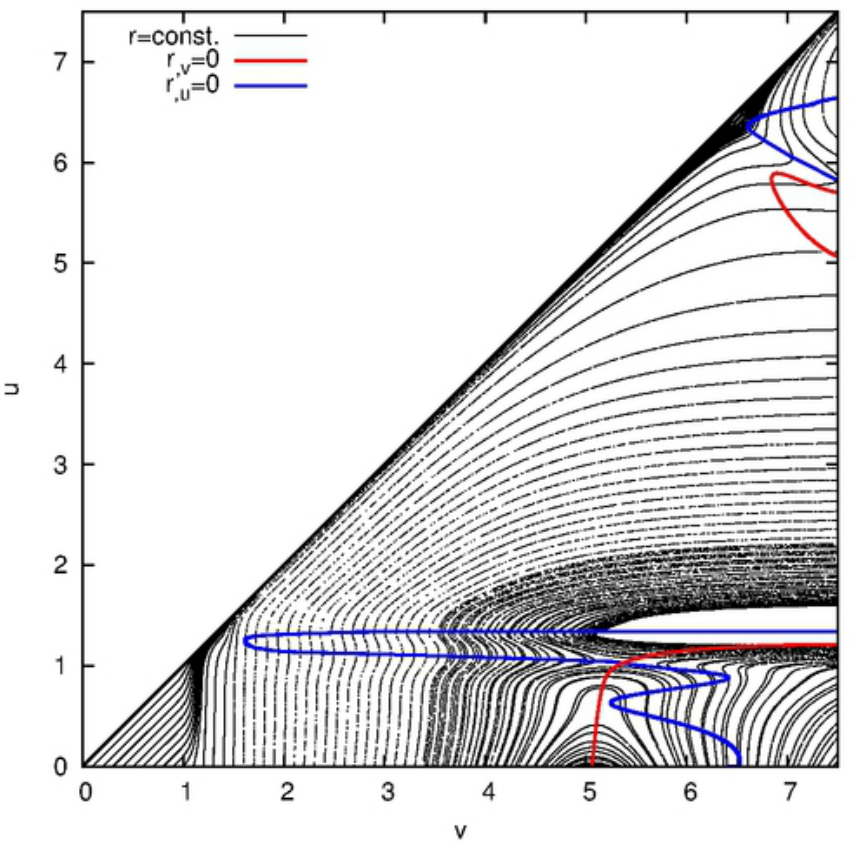

FIG. 8: (color online). Lines of constant $r$ in the $(v u)$-plane for $E M \bar{D}$-evolution with the electric and the dilatonic coupling constants equal to $e=0.5$ and $\alpha=0$, respectively. The family parameter is provided by (a) $\tilde{p}=0.1$, (b) $\tilde{p}=0.8$ and (c) $\tilde{p}=1.1$. 
(a)

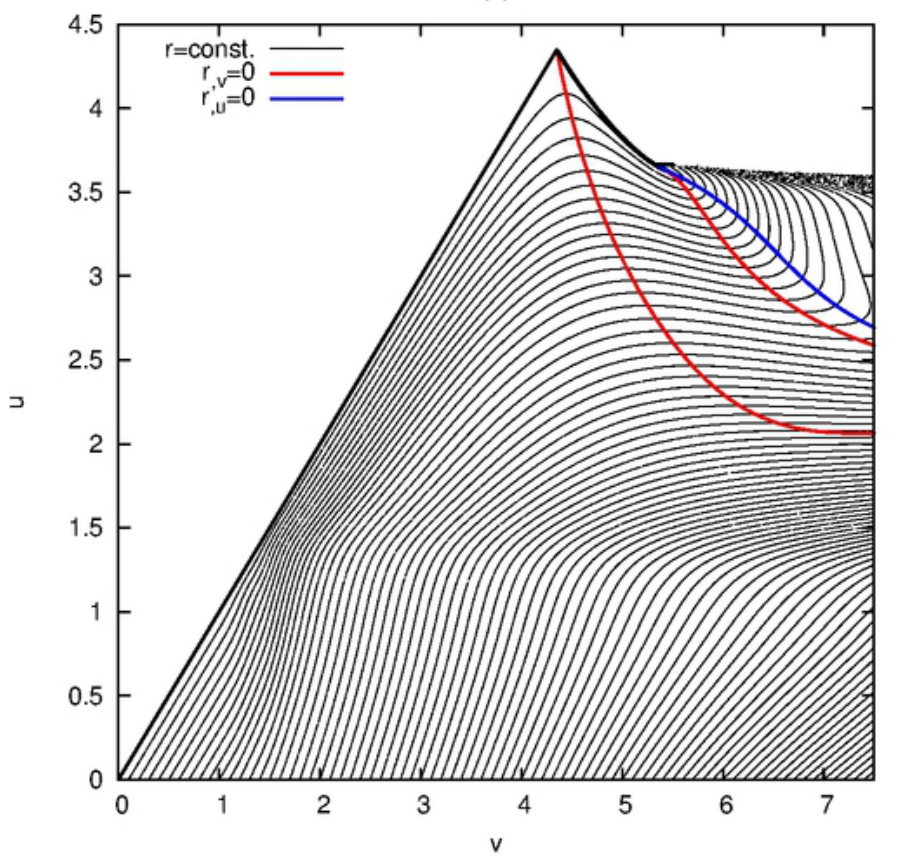

(b)

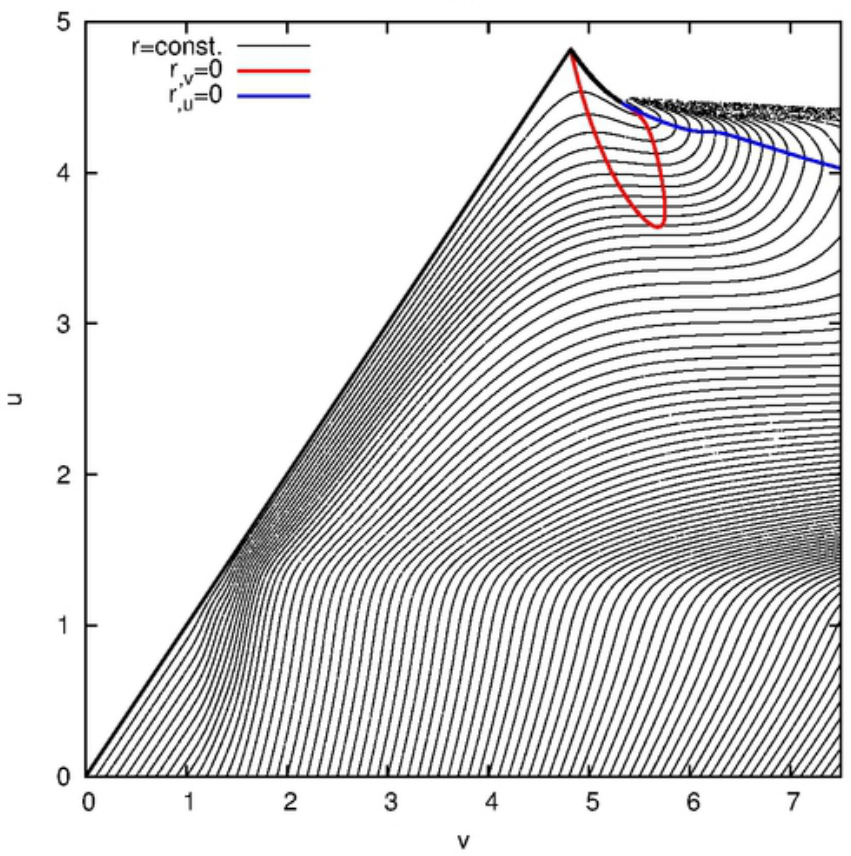

(c)

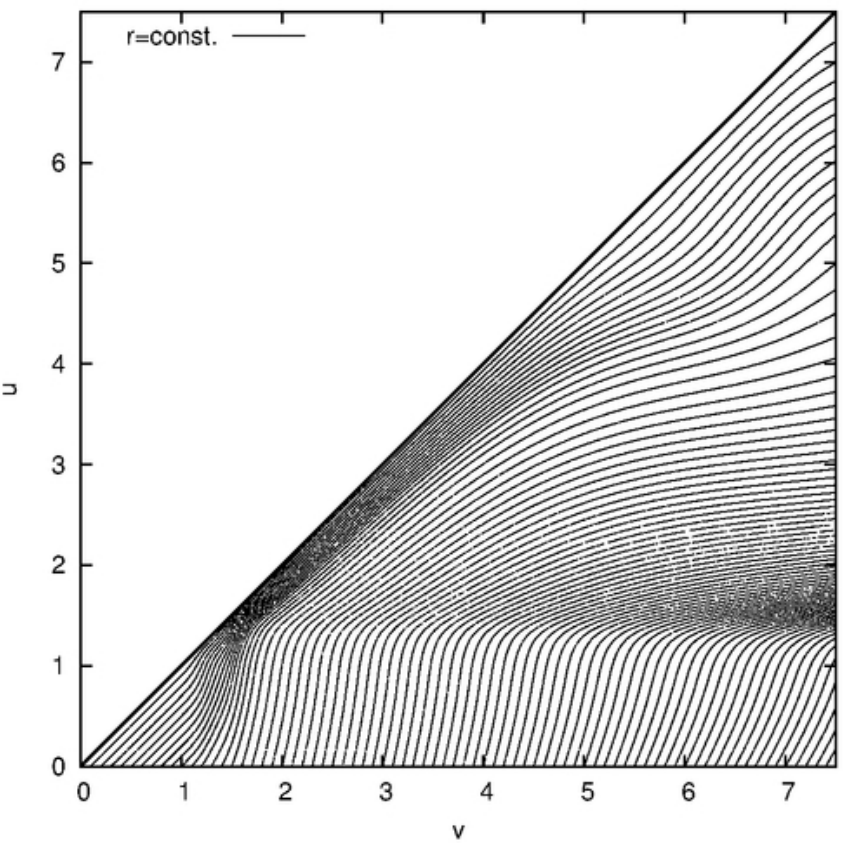

FIG. 9: (color online). Lines of constant $r$ in $(v u)$-plane for $E M \bar{D}$ evolution with a varying dilaton field amplitude. The electric and dilatonic coupling constants are equal to $e=0.5$ and $\alpha=-1$, respectively. The family parameter for the electrically charged scalar field is equal to $\tilde{p}_{s}=0.6$, while for the dilaton field is set as (a) $\tilde{p}_{k}=0.15$, (b) $\tilde{p}_{k}=0.2$ and (c) $\tilde{p}_{k}=0.25$. 
(a)

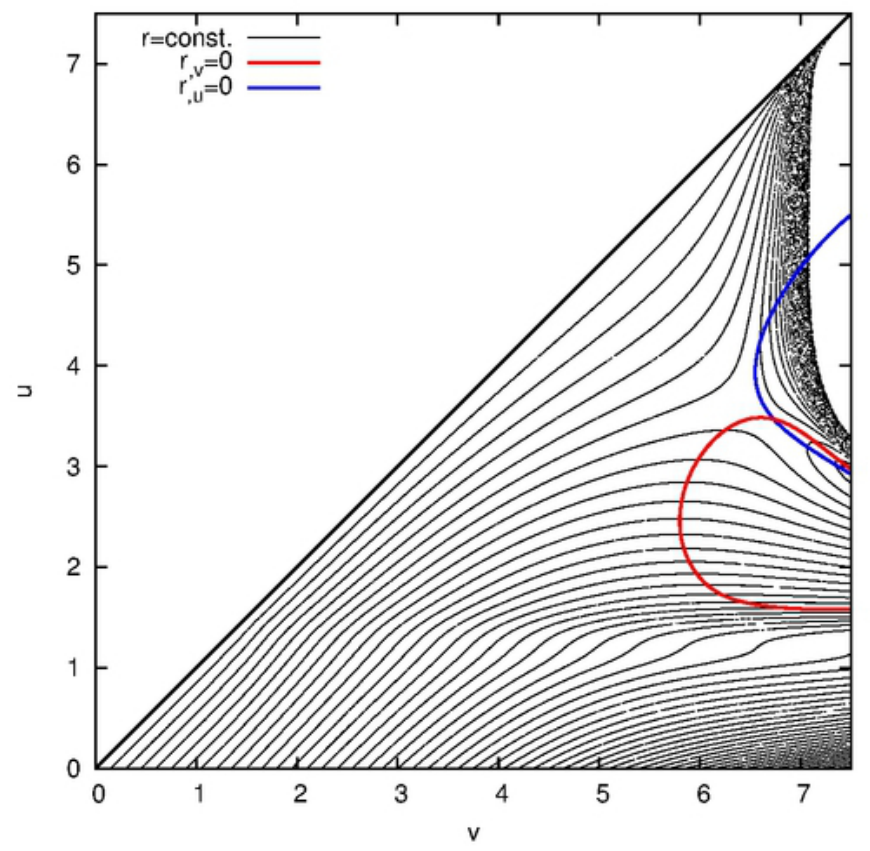

(b)

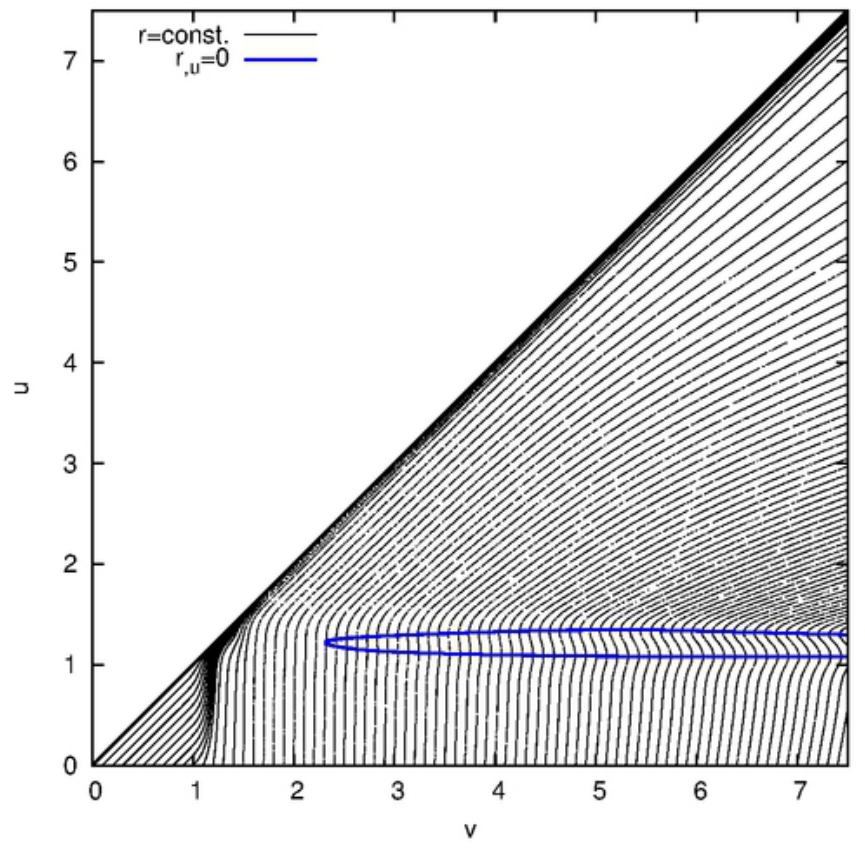

FIG. 10: (color online). Lines of constant $r$ in the $(v u)$-plane for the evolution described in Fig 9 with the dilatonic coupling constant equal to $\alpha=0$ and the family parameter for dilaton field given by (a) $\tilde{p}_{k}=0.05$ and (b) $\tilde{p}_{k}=0.8$. 
(a)

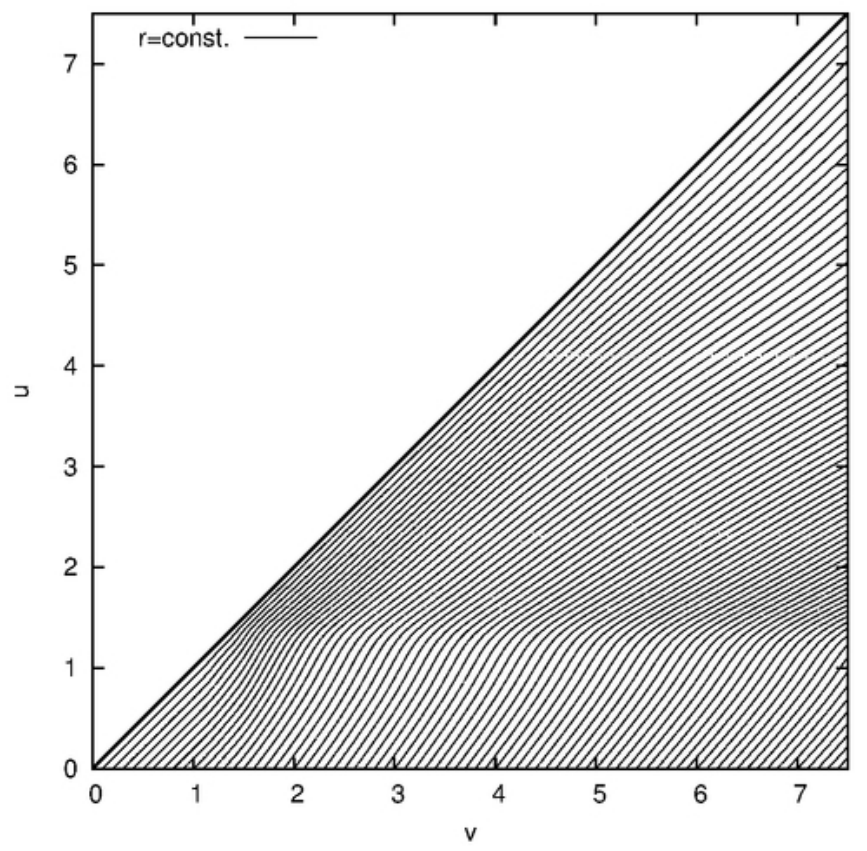

(b)

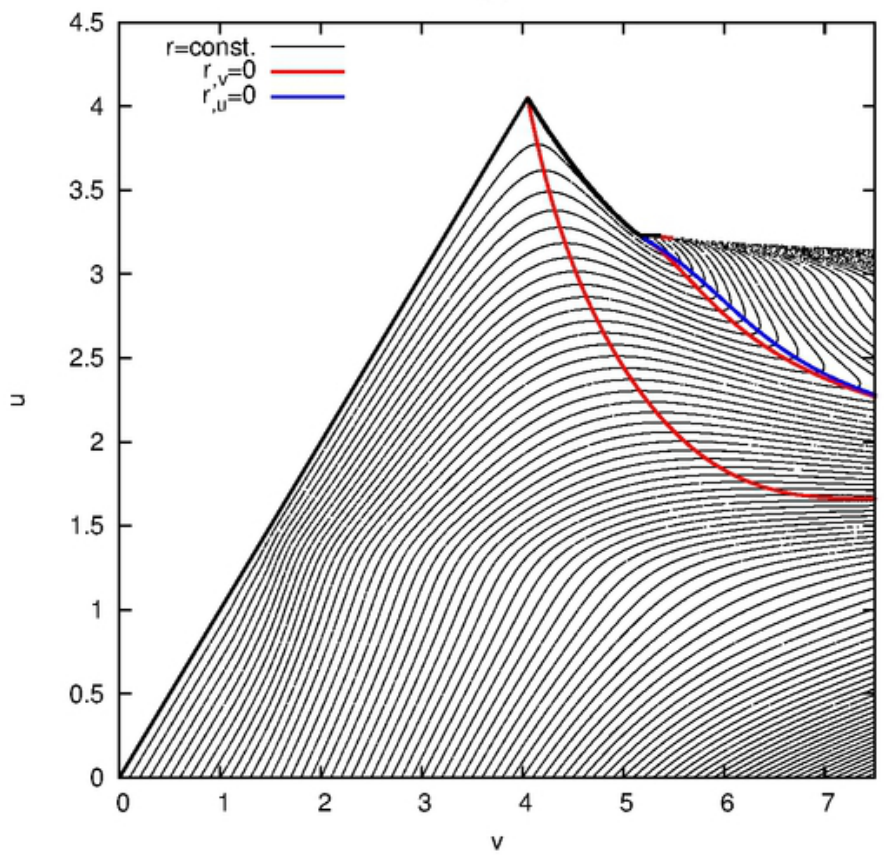

FIG. 11: (color online). Lines of constant $r$ in the $(v u)$-plane for $E M \bar{D}$ evolution with a varying electrically charged scalar field amplitude. The electric and dilatonic coupling constants are equal to $e=0.5$ and $\alpha=-1$, respectively. The family parameter for the dilaton field is $\tilde{p}_{k}=0.1$, while for the electrically charged scalar field field is chosen to be (a) $\tilde{p}_{s}=0.3$ and (b) $\tilde{p}_{s}=0.6$. 
(a)

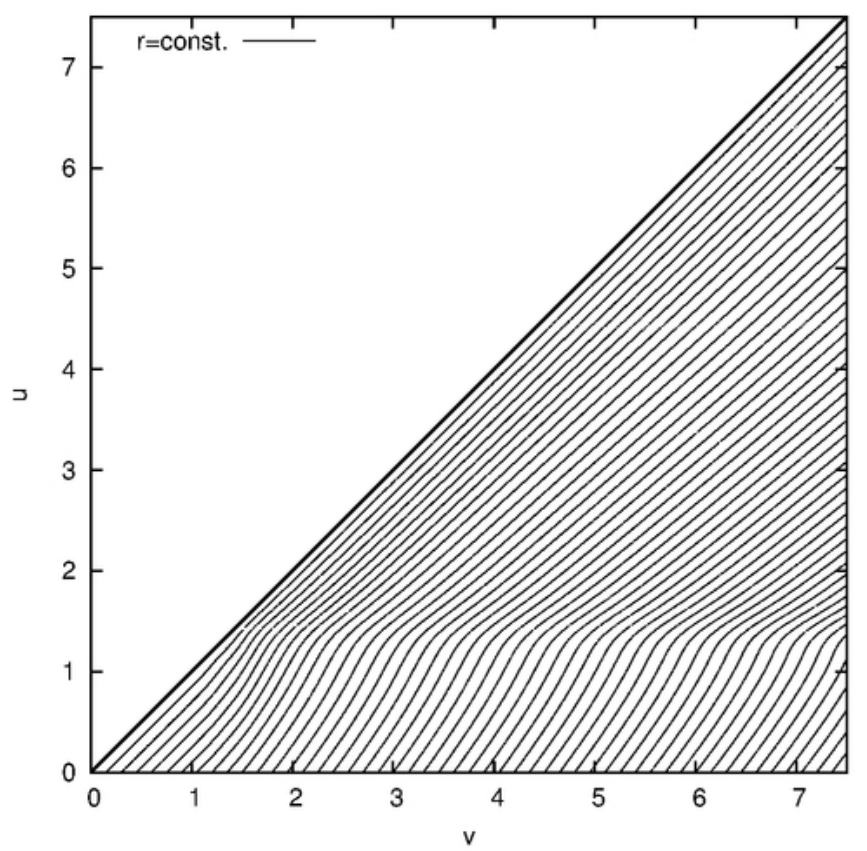

(b)

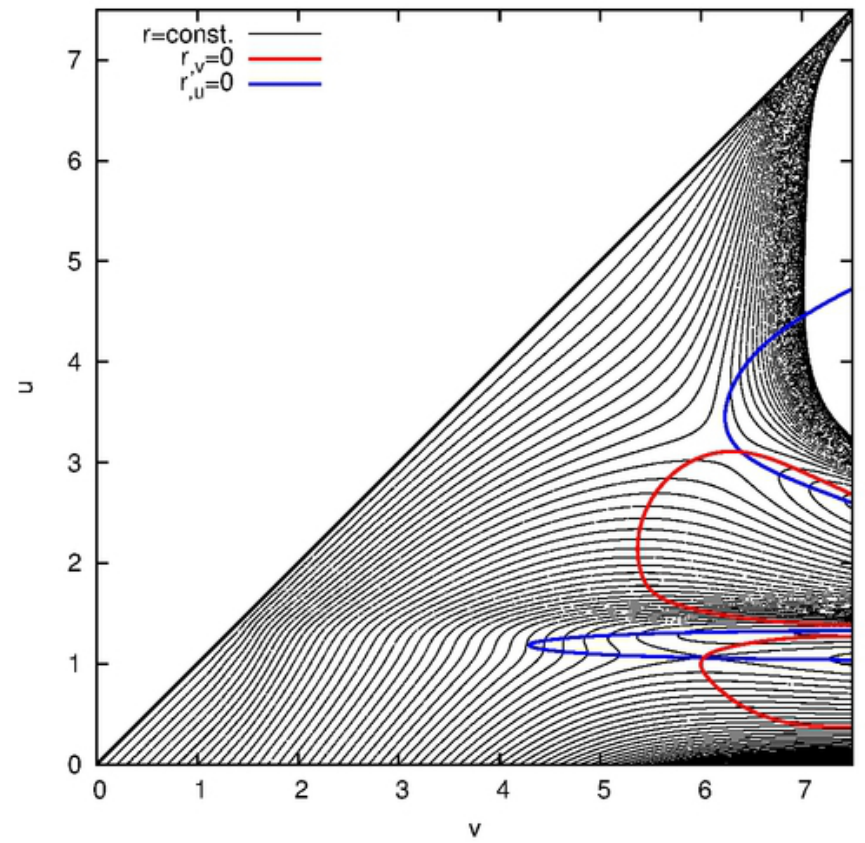

FIG. 12: (color online). Lines of constant $r$ in the $(v u)$-plane for the evolution described in Fig.11 with the dilatonic coupling constant equal to $\alpha=0$ and the family parameter for electrically charged scalar field field (a) $\tilde{p}_{s}=0.2$ and (b) $\tilde{p}_{s}=0.7$. 
(a)

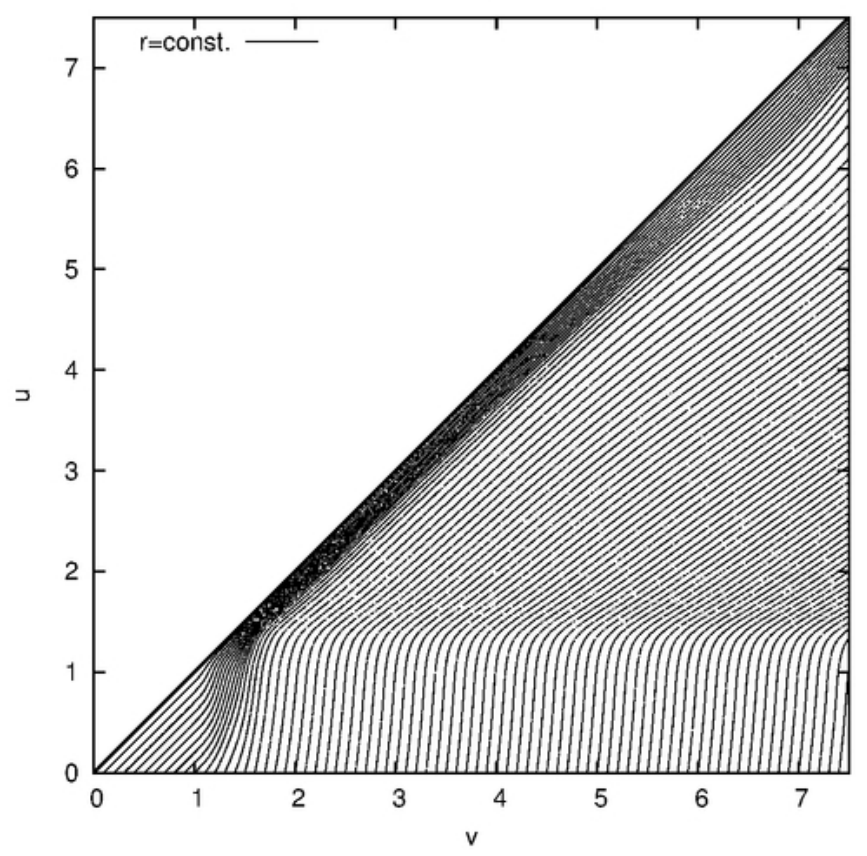

(b)

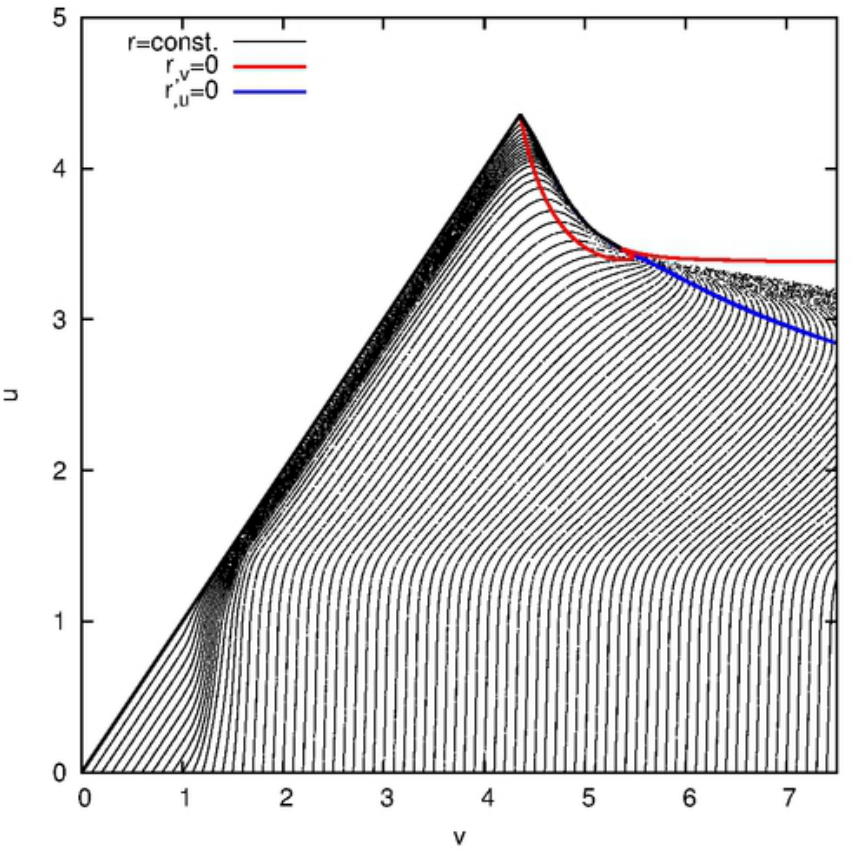

(c)

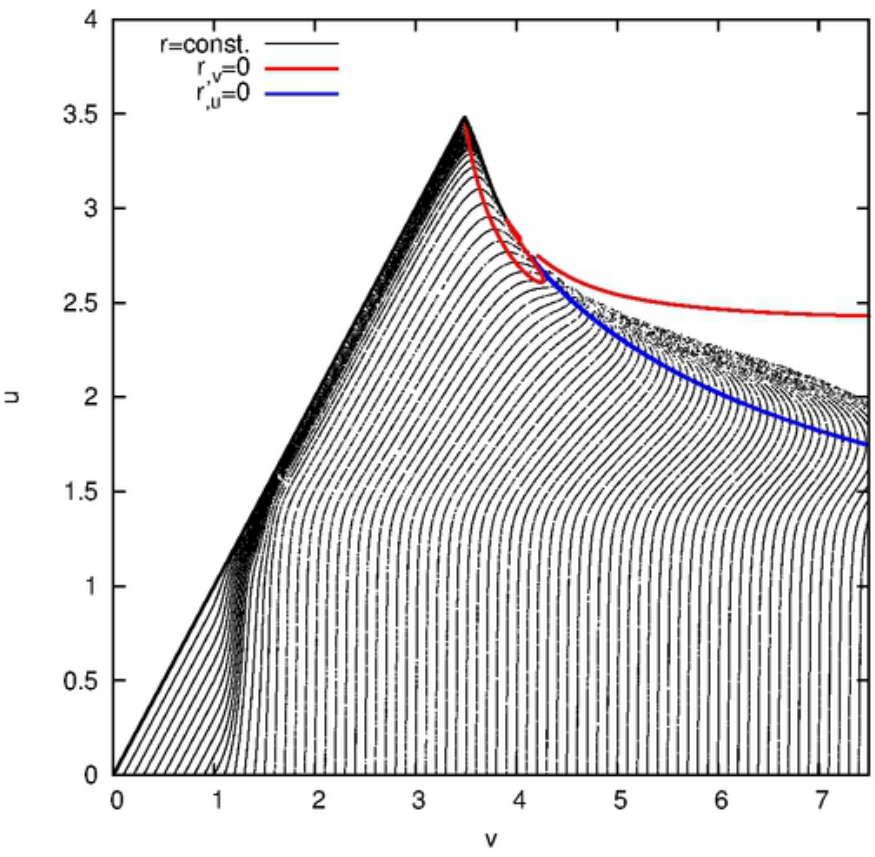

FIG. 13: (color online). Lines of constant $r$ in the $(v u)$-plane for $E \overline{M D}$-evolution with the electric and dilatonic coupling constants equal to $e=0.5$ and $\alpha=-1$, respectively. The family parameter is set (a) $\tilde{p}=0.3$, (b) $\tilde{p}=0.4$ and (c) $\tilde{p}=0.5$. 
(a)

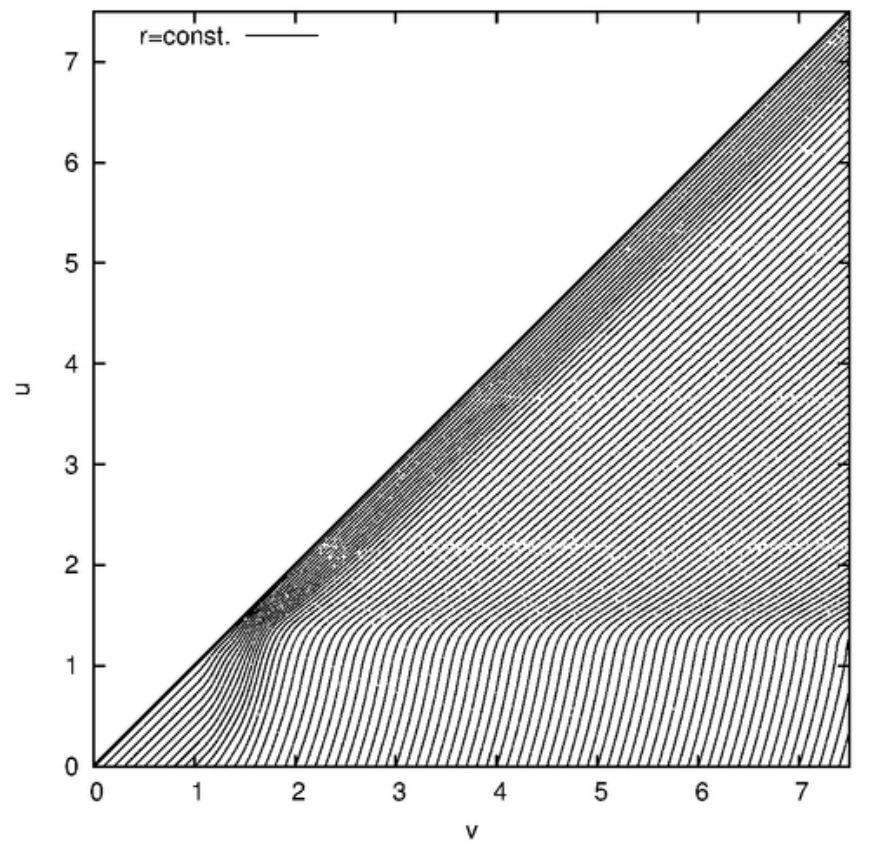

(b)

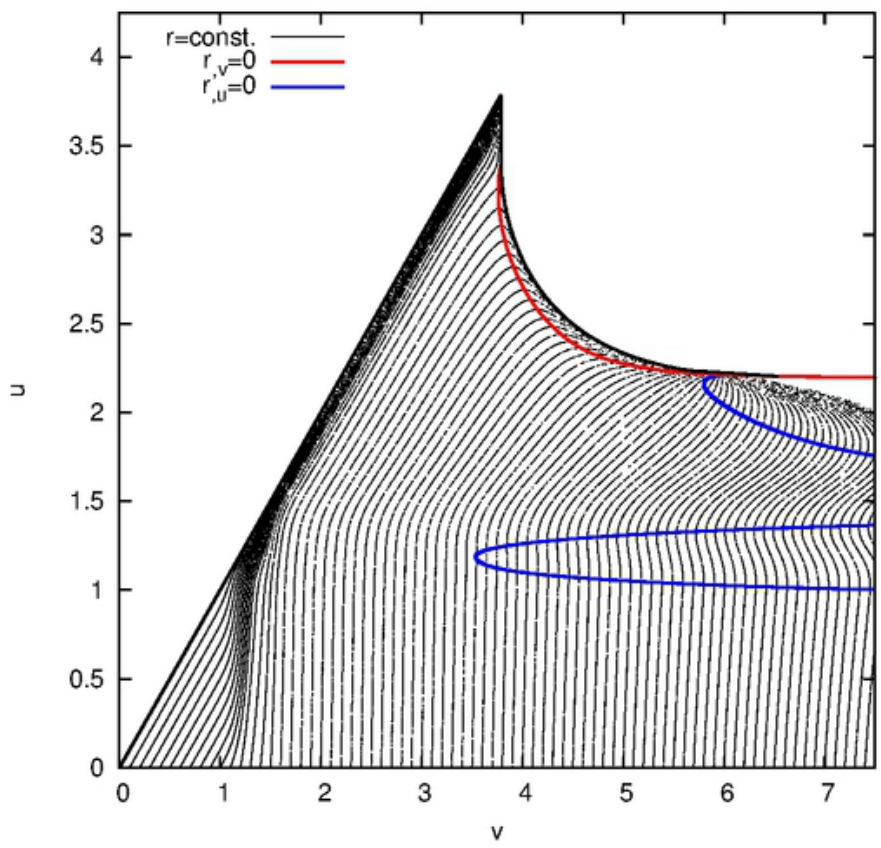

FIG. 14: (color online). Lines of constant $r$ in the $(v u)$-plane for $E \overline{M D}$-evolution with the electric and dilatonic coupling constants equal to $e=0.5$ and $\alpha=0$, respectively. The family p arameter is given by (a) $\tilde{p}=0.2$ and (b) $\tilde{p}=0.5$. 
(a)

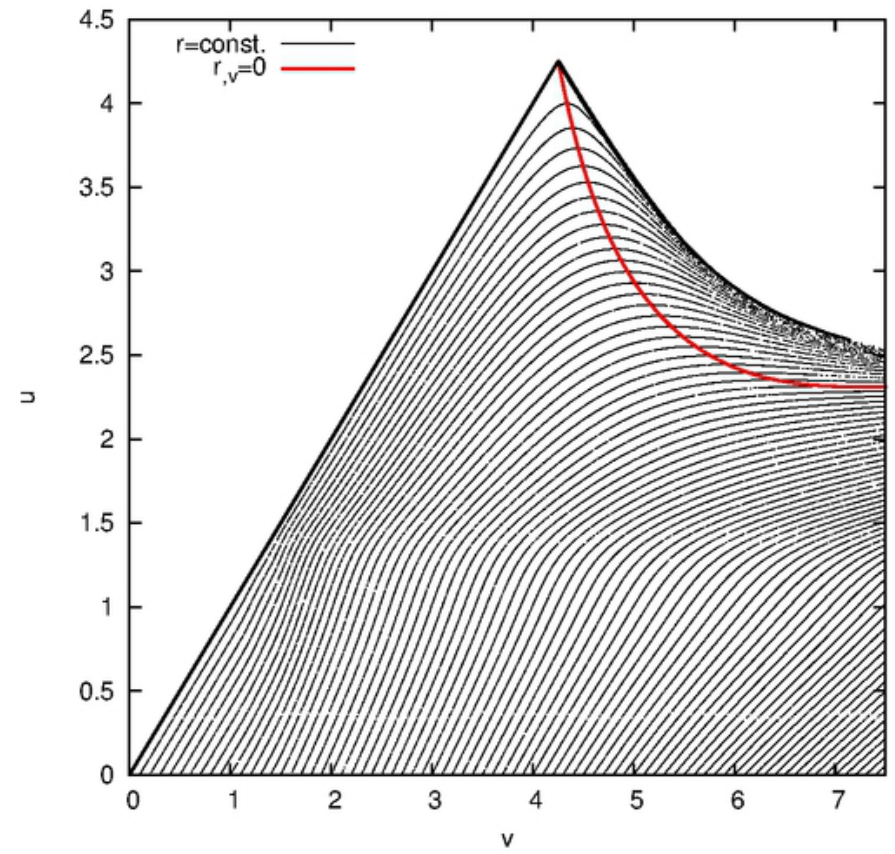

(b)

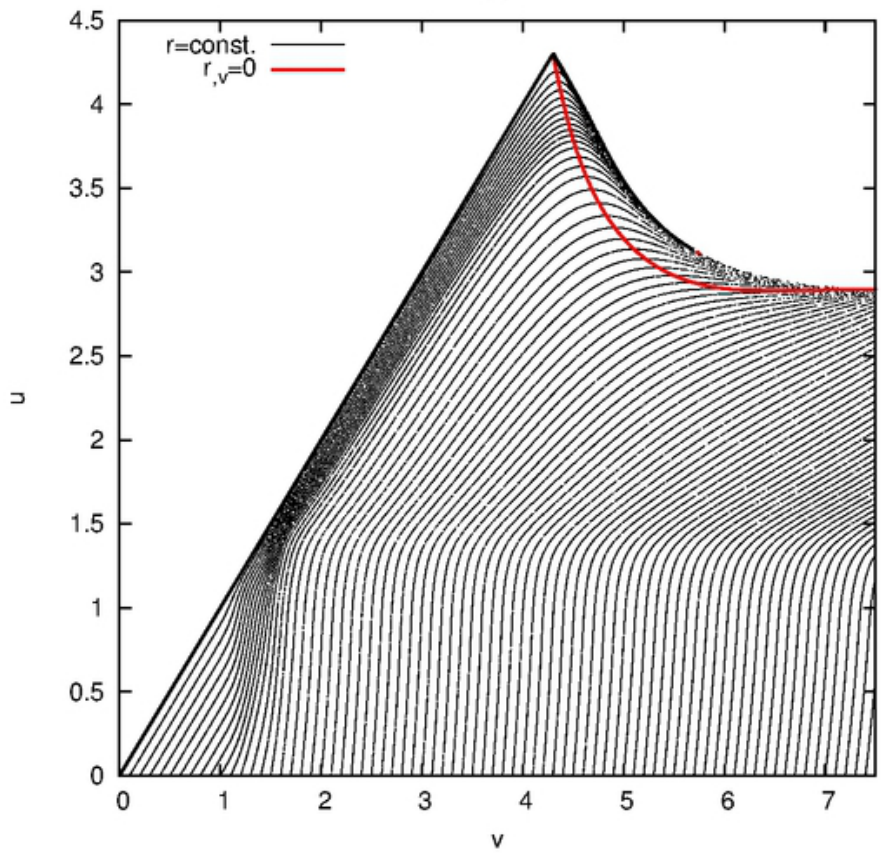

FIG. 15: (color online). Lines of constant $r$ in the $(v u)$-plane for $E \overline{M D}$ evolution with a varying dilaton field amplitude. The electric and dilatonic coupling constants are equal to $e=0.5$ and $\alpha=-1$, respectively. The family parameter for the electrically charged scalar field $\tilde{p}_{s}=0.6$, while for the dilaton field (a) $\tilde{p}_{k}=0.1$ and (b) $\tilde{p}_{k}=0.3$. 
(a)

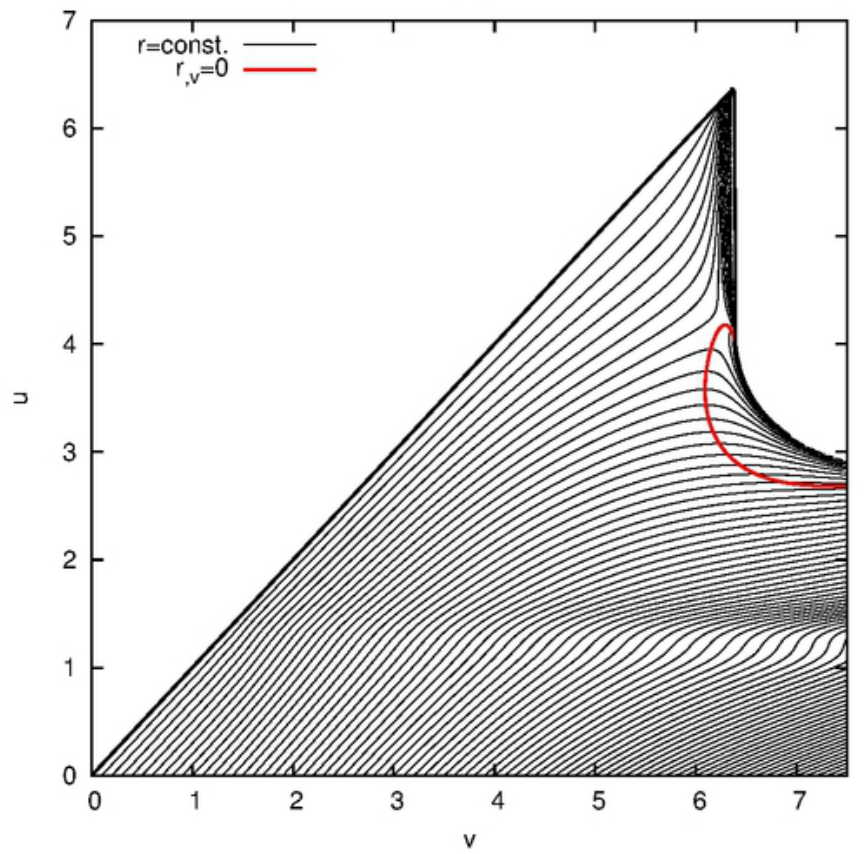

(b)

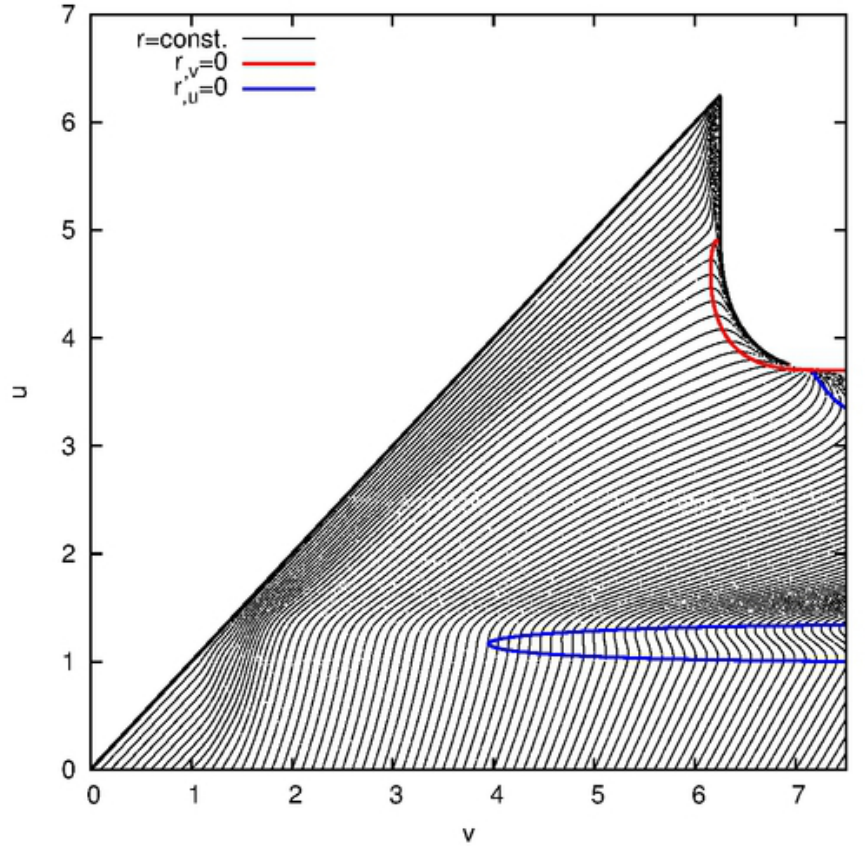

FIG. 16: (color online). Lines of constant $r$ in the $(v u)$-plane for the evolution described in Fig. 15 with the dilatonic coupling constant equal to $\alpha=0$ and the family parameter for dilaton field (a) $\tilde{p}_{k}=0.05$ and (b) $\tilde{p}_{k}=0.2$. 
(a)

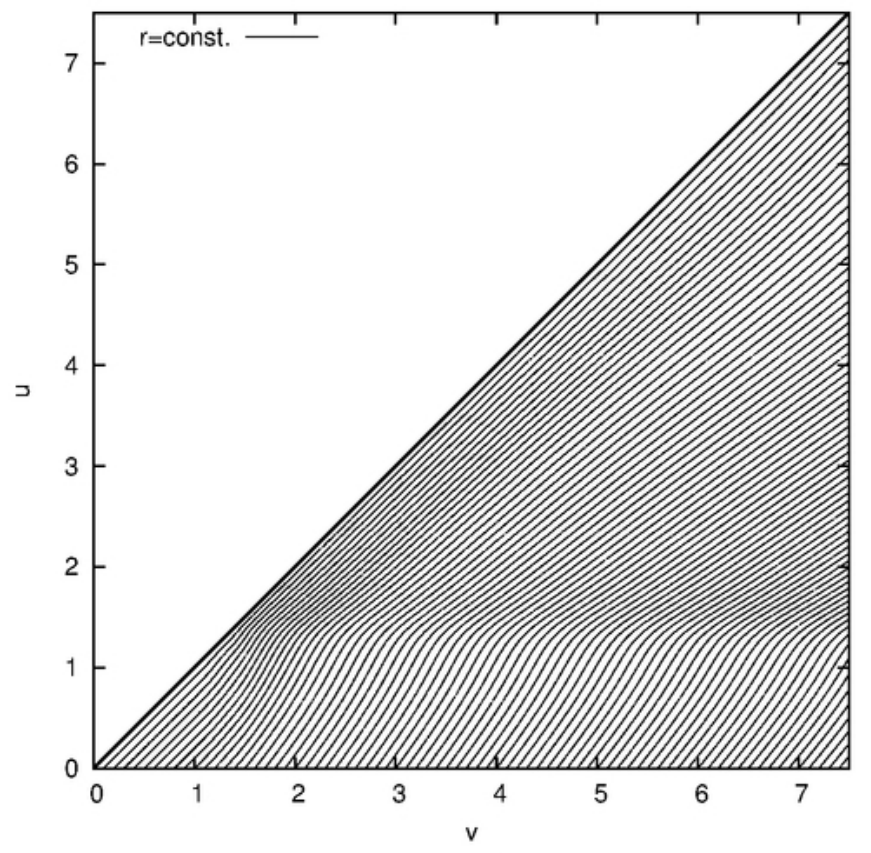

(b)

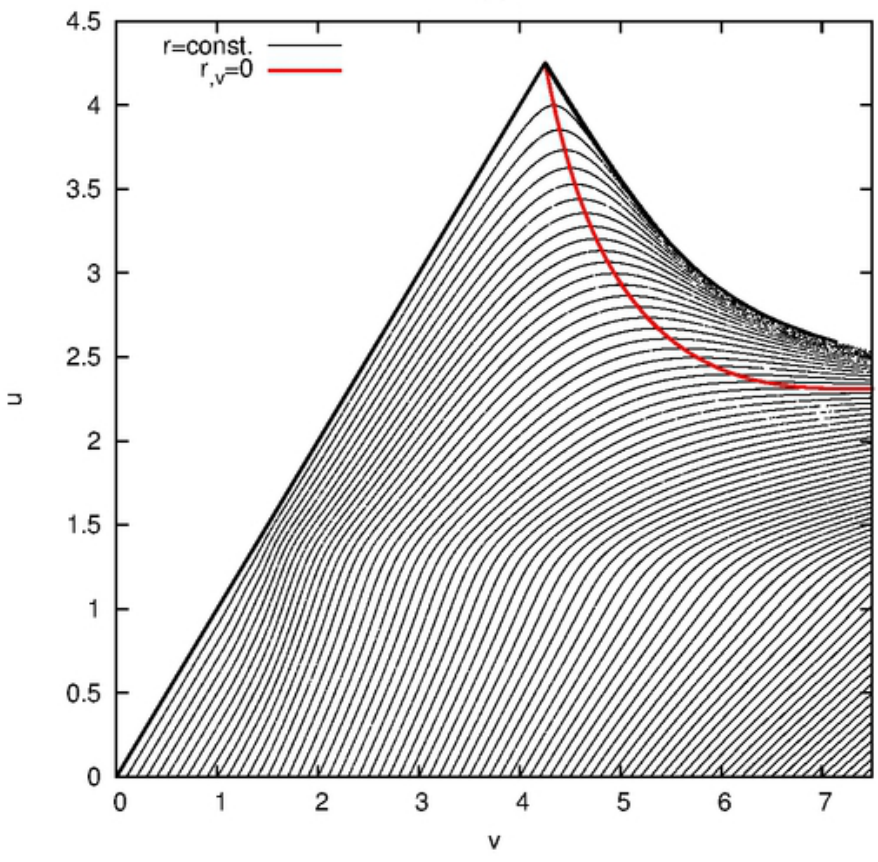

FIG. 17: (color online). Lines of constant $r$ in the $(v u)$-plane for $E \overline{M D}$ evolution with a varying electrically charged scalar field amplitude. The electric and dilatonic coupling constants are equal to $e=0.5$ and $\alpha=-1$, respectively. The family parameter for the dilaton field is chosen as $\tilde{p}_{k}=0.1$, while for the electrically charged scalar field field equals to (a) $\tilde{p}_{s}=0.25$ and (b) $\tilde{p}_{s}=0.5$, respectively. 
(a)

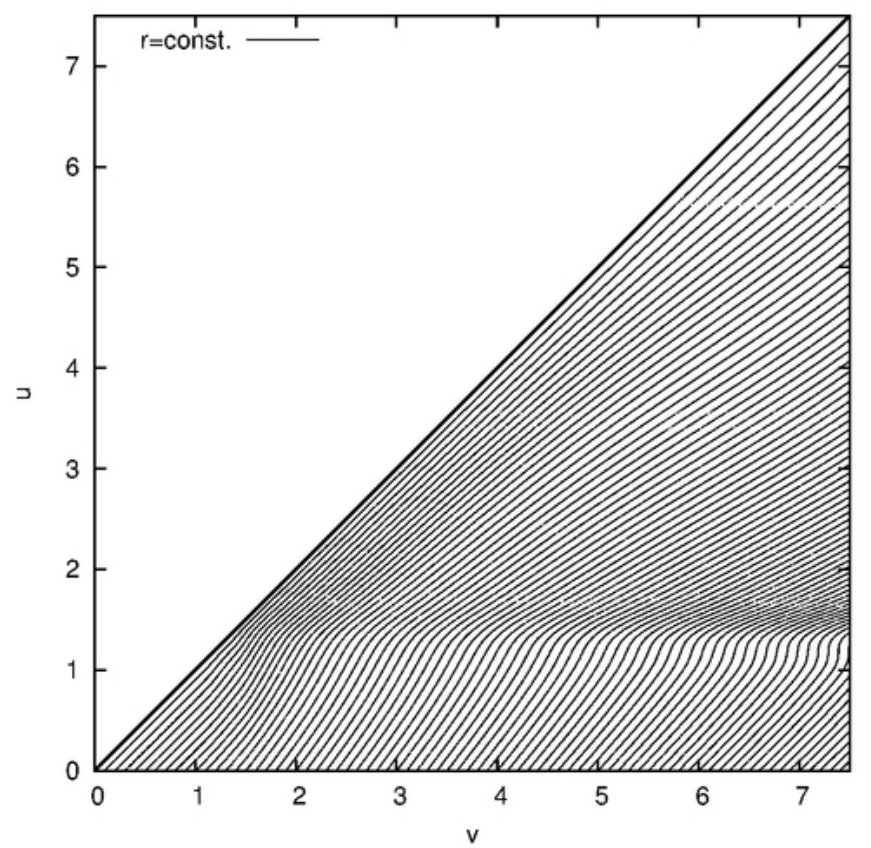

(b)

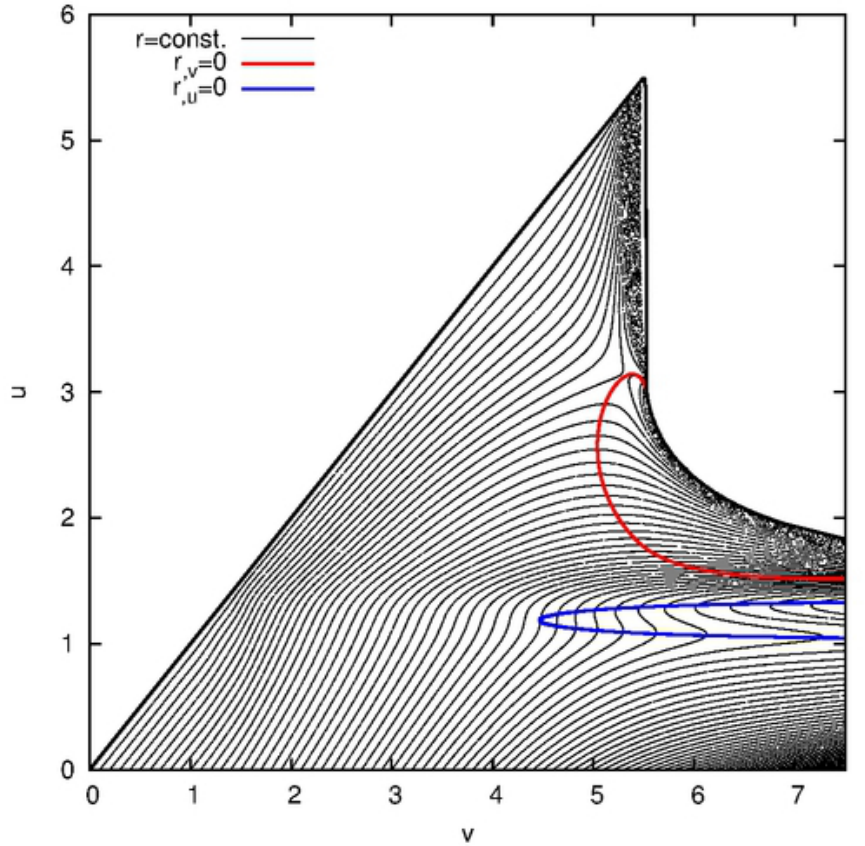

FIG. 18: (color online). Lines of constant $r$ in the $(v u)$-plane for the evolution described in Fig 17 with the dilatonic coupling constant equal to $\alpha=0$ and the family parameter for the electrically charged scalar field field equal to (a) $\tilde{p}_{s}=0.35$ and (b) $\tilde{p}_{s}=0.65$. 